\title{
Seismic attenuation compensation with spectral-shaping regularization
}

\author{
QiZhen Du1,2,3*, WanYu Wang ${ }^{1}$, WenHan Sun ${ }^{4}$, and Li-Yun Fu' ${ }^{1,2,3}$ \\ 'Shandong Provincial Key Laboratory of Deep Oil \& Gas, China University of Petroleum (East China), Qingdao 266580, China; \\ 2Laboratory for Marine Mineral Resources, Qingdao National Laboratory for Marine Science and Technology, Qingdao 266237, China; \\ ${ }^{3}$ Key Laboratory of Geophysical Prospecting, China National Petroleum Corporation (CNPC), China University of Petroleum (East China), \\ Qingdao 266580, China; \\ ${ }^{4}$ Department of Physics, University of Alberta, Edmonton, AB, T6G 2EI, Canada
}

\section{Key Points:}

- We designed an adaptive shaping operator based on the frequency spectra of seismic data and introduced it into inverse-Q filters.

- The data-driven shaping operator can balance the spectral energy distribution of the compensated records through shaping regularization.

- The proposed method can mitigate the relative leakage of low-frequency energy after the high-frequency compensation.

Citation: Du, Q. Z., Wang, W. Y., Sun, W. H., and Fu, L.-Y. (2022). Seismic attenuation compensation with spectral-shaping regularization. Earth Planet. Phys., 6(3), 259-274. http://doi.org/10.26464/epp2022024

\begin{abstract}
Because of the viscoelasticity of the subsurface medium, seismic waves will inherently attenuate during propagation, which lowers the resolution of the acquired seismic records. Inverse-Q filtering, as a typical approach to compensating for seismic attenuation, can efficiently recover high-resolution seismic data from attenuation. Whereas most efforts are focused on compensating for highfrequency energy and improving the stability of amplitude compensation by inverse-Q filtering, low-frequency leakage may occur as the high-frequency component is boosted. In this article, we propose a compensation scheme that promotes the preservation of lowfrequency energy in the seismic data. We constructed an adaptive shaping operator based on spectral-shaping regularization by tailoring the frequency spectra of the seismic data. We then performed inverse-Q filtering in an inversion scheme. This data-driven shaping operator can regularize and balance the spectral-energy distribution for the compensated records and can maintain the low-frequency ratio by constraining the overcompensation for high-frequency energy. Synthetic tests and applications on prestack common-reflectionpoint gathers indicated that the proposed method can preserve the relative energy of low-frequency components while fulfilling stable high-frequency compensation.
\end{abstract}

Keywords: seismic attenuation compensation; spectral-shaping regularization; data-driven shaping operator; offset-related inverse-Q filter

\section{Introduction}

Because of the inherent absorption of the subsurface medium, seismic waves will inevitably be influenced by amplitude decays and phase distortions during propagation. These changes, known as seismic attenuation (Zhang BL et al., 2019), are typically characterized by the quality factor $\mathrm{Q}$ and will contaminate the quality of the acquired data. The mechanisms of seismic wave attenuation have been widely studied since the last century (Biot, 1956; Chernov, 1960; Mavko and Nur, 1975). Seismic wave propagation in a viscoelastic medium is one significant theory used to characterize subsurface attenuation, and several well-known models have been proposed and widely applied using this framework

Correspondence to: Q. Z. Du, multicomponent@163.com

Received 25 NOV 2021; Accepted 04 MAR 2022.

Accepted article online 24 MAR 2022.

C 2022 by Earth and Planetary Physics.
(Kolsky, 1953, 1956; Futterman, 1962; Kjartansson, 1979). From these models, various state-of-the-art compensation methods have been developed in an attempt to eliminate the attenuation effects.

As a common approach to compensating for seismic attenuation, inverse-Q filtering has been widely used for resolution enhancement. The various techniques of inverse- $Q$ filtering can be classified into two general categories according to the direction of wavefield continuation. One category utilizes backward wavefield continuation to directly compensate for attenuation. Hale (1981, 1982) proposed Q-adaptive deconvolution to implement high-frequency compensation for the seismic signal. The method generated critical insights for the studies that followed (Bickel and Natarajan, 1985; Varela et al., 1993). Hargreaves and Calvert (1991) applied inverse-Q filtering with reference to Stolt frequencywavenumber migration (Stolt, 1978) to correct phase distortions 
in the seismic records. Wang YH (2002) further achieved amplitude compensation with this method by incorporating a stabilization factor to avoid numerical instability. This migration-based method has resulted in notable developments and applications over the past two decades (Wang YH, 2003, 2006; Rao Y and Wang $\mathrm{YH}, 2019)$. Margrave et al. $(2002,2011)$ proposed a Gabor deconvolution based on a nonstationary convolution model and realized attenuation compensation in the time-frequency domain. In addition, other time-frequency techniques, such as the $S$ transform (Wang BF and Lu WK, 2018) and the synchro-squeezing transform (Xue YJ et al., 2019), were attempted to compensate for seismic attenuation. The other category of inverse- $Q$ filtering, which is based on forward wavefield continuation and regards compensation as an inverse problem, has received increasing attention partly because of its robustness to amplitude compensation. Zhang CJ and Ulrych (2007) utilized the hypothesis of sparse reflection coefficients to construct a Bayesian optimization model, through which seismic data were stably compensated by a leastsquares inversion. Regularization techniques have also been investigated in inversion-based inverse- $Q$ filtering to enforce the smoothness (Wang SD, 2011; Wang BF et al., 2015) and sparsity (Wang SD and Chen XH, 2014; Wang YF et al., 2018) of the solutions. In recent years, more high-resolution computational strategies have been evaluated for their potential applications in inverse-Q filtering (Yuan SY et al., 2017; Zhang GW and Gao JH, 2018).

As for prestack seismic data, the propagation paths of seismic waves vary with offsets, and trace-variant (offset-related) attenuation needs to be considered in compensation. Q-compensated prestack migration offers an accurate approach that compensates for seismic attenuation along the wavefield propagation paths (Mittet et al., 1995; Wang YH and Guo J, 2004b). Ray-based migration methods generally introduce an absorption-dependent travel time to achieve efficient compensation for the waveforms (Zhang JF and Wapenaar, 2002; Traynin et al., 2008; Xie Y et al., 2009; Zhang JF et al., 2013). Reverse-time migration, as a representative wave-equation migration approach, has the potential to accurately image steep discontinuities and complex geological structures (Chang WF and McMechan, 1994; Du QZ et al., 2012, 2017; Liu YS et al, 2019). Various studies have incorporated Q-compensation into reverse-time migration, with the Kjartansson constant $Q$ theory (Carcione et al., 2002; Treeby and Cox, 2010; Zhang Y et al., 2010; Zhu TY et al., 2014; Sun JZ et al., 2015) and the generalized standard linear solid theory (Carcione et al., 1988; Robertsson et al., 1994; Blanch et al., 1995; Deng F and McMechan, 2007) being the two primary mechanisms used for $Q$ parameterization (Guo $P$ et al., 2016). Q-compensation has also been investigated in Gaussian beam migration (Bai M et al., 2016; Shi XC et al., 2019), which provides a competitive alternative that balances the computational costs with the precision of migration. Compared with the computationally intensive migration techniques, prestack-oriented inverse- $Q$ filtering offers a less expensive way to correct for the amplitude loss and phase distortions across offsets. For instance, Braga and Moraes (2013) proposed an inverse-Q filter based on a wavelet transform and applied it on prestack gathers. Although the impacts of offsets were not considered, the amplitude variation with offset information was preserved well after compensation. Yan HY and Liu Y (2009) extended the stabilized inverse-Q filtering to prestack seismic data and estimated travel time through a ray-tracing technique. Li GF et al. (2015) decomposed attenuation into time and offset components, then applied a two-step scheme for the compensation of prestack gathers.

Q estimation is another critical issue that determines the performance of attenuation compensation (Wang, 2008). Studies of raybased $Q$ estimation, which date back to the last century, measure attenuation from either waveform amplitudes (Brzostowski and McMechan, 1992) or amplitude spectra (e.g., Tonn, 1991; Quan YL and Harris, 1997; Dasgupta and Clark, 1998; Wang YH, 2004) to build full Q tomographic images or local Q models. Because of the lack of theories that deal with the multipathing of the reflected energy, the ray-based method often fails to generate a reliable $Q$ estimate in complex geological contexts (Shen Y et al., 2018). A wave-equation-based $\mathrm{Q}$ estimation provides an accurate way to characterize wave propagation, but at the expense of much higher computational costs. Wave-equation methods are generally performed in an iterative scheme, in which $\mathrm{Q}$ models are updated by minimizing the misfit functions of the spectral changes (Dutta and Schuster, 2016), waveform residuals (e.g., Liao OB and McMechan, 1995; Kamei and Pratt, 2008, 2013; Bai T et al., 2017), or residual images (Shen $Y$ et al., 2014, 2018). Despite the great efforts expended on $Q$ estimation, obtaining a reliable $Q$ estimate remains a difficult task because of the complexity of the wavefield in relation to the assumptions that facilitate numerical computation. An incorrect estimate from a $\mathrm{Q}$ model as the input may lead to corruption in the $\mathrm{Q}$ compensation. Typically, compensation using $Q$ values that are too small can overboost the high-frequency energy, whereas the ratio of low-frequency energy across the frequency spectra will be relatively reduced.

In this study, we designed an adaptive shaping operator to perform spectral shaping on the inversion-based compensation through shaping regularization (Fomel, 2007). Chen SQ et al. (2018) investigated the scheme of attenuation compensation via shaping regularization and designed an adaptive tapering window to improve the robustness to noise. In contrast, we explored the spectral-shaping capability of this regularization scheme by designing a shaping operator based on the frequency spectra of the seismic data. The data-driven operator plays a role in shaping and balancing the spectra of the compensated data. This spectralshaping-regularized (SSR) compensation has the potential to maintain low-frequency energy more efficiently while also achieving a stable recovery of high-frequency energy. This article is organized in the following manner. We first briefly introduce the offset-related $\mathrm{Q}$ filters, which are based on a modified Kolsky model. We then discuss the inversion-based compensation with shaping regularization. Following discussion of the shaping regularization, we further describe the calculations of the proposed spectralshaping operator. Finally, we use synthetic models and actual data to verify the spectral-shaping performance of the proposed 
shaping operator.

\section{Theory and Method}

\subsection{Offset-Related Q Filters with Effective-Q Approximation}

Suppose that the subsurface medium is horizontally stratified, as shown in Figure 1. The thickness, velocity, and quality factor of each layer $i$ are denoted as $h_{i}, \boldsymbol{v}_{i}$, and $Q_{i}$. The black arrows schematically show one propagation path of seismic waves, with $\theta_{i}$ representing incident angles at each interface. On such occasions, we can obtain the inverse- and forward-Q filters that vary with offset. In this work, we utilized a modified Kolsky model (Wang YH and Guo J, 2004a) to construct the Q filters (see the Appendix for the detailed derivation). $\mathrm{Q}$ filtering can be implemented with a matrix computation (Hargreaves and Calvert, 1991) as

$$
u_{N \times 1}=A_{N \times M}^{d} U_{M \times 1}
$$

where $\boldsymbol{u}_{N \times 1}, \boldsymbol{A}_{N \times M^{\prime}}^{d}$ and $\boldsymbol{U}_{M \times 1}$ are, respectively, the compensated signal, $Q$ filtering operator, and frequency spectrum of the uncompensated signal. Here, $d=1$ for forward-Q filtering or $d=-1$ for inverse- $Q$ filtering, and $M$ and $N$ are the sampling numbers in the frequency domain and the time domain. Equation (1) can be expanded as

$$
\left(\begin{array}{c}
u_{1} \\
\ldots \\
u_{n} \\
\ldots \\
u_{N}
\end{array}\right)_{N \times 1}=\left(\begin{array}{ccccc}
a_{11}^{d} & \ldots & a_{1 m}^{d} & \ldots & a_{1 M}^{d} \\
\ldots & \ldots & & & \\
a_{n 1}^{d} & & a_{n m}^{d} & & \\
\ldots & & & \ldots & \\
a_{N 1}^{d} & & & & a_{N M}^{d}
\end{array}\right)_{N \times M}\left(\begin{array}{c}
U_{1} \\
\ldots \\
U_{m} \\
\ldots \\
U_{M}
\end{array}\right)_{M \times 1}
$$

where $u_{n}, a_{n m}^{d}$, and $U_{m}$ are the elements in $\boldsymbol{u}_{N \times 1}, \boldsymbol{A}_{N \times M}^{d}$, and $\boldsymbol{U}_{M \times 1}$, respectively. When $d=-1$, element $a_{n m}^{-1}$ in the inverse-Q operator $\boldsymbol{A}_{N \times M}^{-1}$ is

$$
\left.a_{n m}^{-1}=\frac{1}{M} e^{j\left[\omega_{m}\left(\frac{\omega_{m}}{\omega_{h}}\right)^{-\gamma e, n}\right.} \sum_{i=1}^{n} \frac{\mathrm{d} t}{\cos \theta_{i}}\right] \mathrm{e}\left[\left(\frac{\omega_{m}}{\omega_{h}}\right)^{-\gamma_{e, n}} \frac{\omega_{m}}{2 Q_{e, n}} \sum_{i=1}^{n} \frac{\mathrm{d} t}{\cos \theta_{i}}\right],
$$

where $\mathrm{d} t$ and $\omega_{m}$ denote the sampling interval and discrete frequency, respectively. The discrete frequency $\omega_{h}$ is the tuning frequency, $Q_{e, n}$ represents the effective quality factor, and

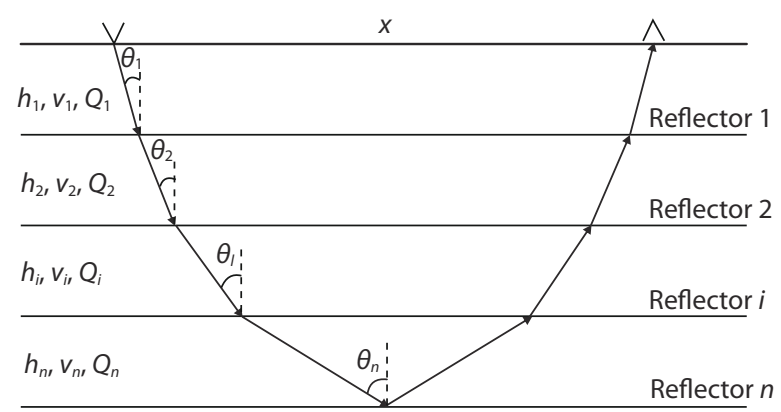

Figure 1. Sketch map of seismic ray propagation (black arrows) in a horizontally stratified medium, where is the offset between simulated source and receiver. The strata are labeled with numbers $1,2, \cdots, i, \cdots, n$. Parameters $h_{i}, v_{i}$, and $Q_{i}$ respectively denote the thickness, velocity, and quality factor of each layer, and $\theta_{i}$ is the incident angle of rays at each reflector. $\gamma_{e, n}=\frac{1}{\pi} \tan ^{-1} \frac{1}{Q_{e, n}} \approx \frac{1}{\pi Q_{e, n}}$ (Kjartansson, 1979). Equation (3) illustrates the numerical computation of offset-related inverse-Q filtering. The first exponential term is used for phase corrections, whereas the second term functions to compensate for the amplitude but is subject to inherent instability. Therefore, the inverse-Q filters are normally implemented by using a more stable scheme (Wang $\mathrm{YH}$, 2002),

$$
\begin{gathered}
a_{n m}^{-1} \approx \frac{1}{M} \frac{\Lambda_{n m}+\sigma^{2}}{\Lambda_{n m}^{2}+\sigma^{2}} e^{j\left[\omega_{m}\left(\frac{\omega_{m}}{\omega_{h}}\right)^{-\gamma_{e, n}} \sum_{i=1}^{n} \frac{\mathrm{d} t}{\cos \theta_{i}}\right],} \\
\Lambda_{n m}=\mathrm{e}^{-\left[\left(\frac{\omega_{m}}{\omega_{h}}\right)^{-\gamma_{e, n}} \frac{\omega_{m}}{2 Q_{e, n}} \sum_{i=1}^{n} \frac{\mathrm{d} t}{\cos \theta_{i}}\right],}
\end{gathered}
$$

in which $\sigma$ is the stabilization factor that stabilizes the amplitude compensation.

When $d=1$, the forward-Q operator $\boldsymbol{A}_{N \times M}^{1}$ performs signal attenuation. We simplify it as $\boldsymbol{A}_{N \times M}$, and its element $a_{n m}$ (Wang YH, 2008) can be expressed as

$$
a_{n m} \approx \frac{1}{M} e^{j\left[\omega_{m}\left(\frac{\omega_{m}}{\omega_{h}}\right)^{\gamma e, n} \sum_{i=1}^{n} \frac{\mathrm{d} t}{\cos \theta_{i}}\right]} \mathrm{e}^{-\left[\left(\frac{\omega_{m}}{\omega_{h}}\right)^{-\gamma_{e, n}} \frac{\omega_{m}}{2 Q_{e, n}} \sum_{i=1}^{n} \frac{\mathrm{d} t}{\cos \theta_{i}}\right] .}
$$

\subsection{Inversion-based Compensation with Spectral-Shaping Regularization}

Inversion-based compensation utilizes the physical process of seismic attenuation and regards compensation as an inverse problem to guarantee the numerical stability of amplitude compensation. The Tikhonov approach (Tikhonov, 1963), which is based on the zero-offset forward-Q filter, involves implementing a quadratic regularization (Wang BF et al., 2015),

$$
\boldsymbol{U}=\min _{\boldsymbol{U}}\|\boldsymbol{A U}-\boldsymbol{u}\|_{2}^{2}+\lambda\|D U\|_{2}^{2}
$$

where $\boldsymbol{A}, \boldsymbol{U}$, and $\boldsymbol{u}$ have the same physical meanings as in Equation (1) when $d=1$. The compensation is regularized by operator $D$, with $\lambda$ denoting the regularization factor. The analytical solution of this cost function is $U=\left(A^{\top} A+\lambda D^{\top} D\right)^{-1} A^{\top} u$.

Chen SQ et al. (2018) introduced shaping regularization (Fomel, 2007) into the attenuation compensation and demonstrated its robustness to noise. The formal solution of the shaping-regularized inversion originates from the well-known solution of the Tikhonov approach, as shown above, while replacing the operator $\boldsymbol{D}$ by an operator $S=\left(I+\lambda D^{\top} D\right)^{-1}$ and

$$
\boldsymbol{U}=\left[\mu^{2} \boldsymbol{I}+\boldsymbol{S}\left(\boldsymbol{A}^{\top} \boldsymbol{A}-\mu^{2} \boldsymbol{I}\right)\right]^{-1} \boldsymbol{S} \boldsymbol{A}^{\top} \boldsymbol{u} .
$$

Operator $S$ functions as a shaping operator that translates the input into an expected model (Fomel, 2007). Factor $\mu$ is a scaling factor that controls the relative weights between the forward- $Q$ filtering operator $\boldsymbol{A}$ and the shaping operator $\boldsymbol{S}$. A larger $\mu$ will result in a stronger shaping performance. Matrix / denotes the identity matrix.

\subsection{An Adaptive Spectral-Shaping Operator}

The accurate estimation of $Q$ can be difficult in field cases. The expression of the inverse- $Q$ filter establishes that the compensation is intensified exponentially with increasing frequency. Consequently, unsuitable selections of $Q$ models may lead to excess- 
ive high-frequency compensation for the seismic data, which leads to a relative loss of low-frequency energy. In this study, we explored the spectral-shaping performance of the shaping regularization to impose a constraint that promotes the preservation of low-frequency energy in the inversion-based compensation. Specifically, we designed a data-driven shaping operator that could be adaptively obtained from the seismic records,

$$
\begin{gathered}
S=\operatorname{diag}\left(\widehat{\boldsymbol{w i n}^{S}}\right), \\
\boldsymbol{w i n}^{S}=\widehat{\boldsymbol{w i n}^{\alpha}} \cdot \boldsymbol{w i n}^{\beta},
\end{gathered}
$$

where $\operatorname{diag}(\cdot)$ denotes vector diagonalization and $\widehat{(\cdot)}$ is the normalization operator. Shaping window $\operatorname{win}^{S}$ is the multiplication of $\boldsymbol{w i n}^{\alpha}$ and $\boldsymbol{w i n}^{\beta}$, which are

$$
\begin{gathered}
\boldsymbol{w i n}^{\alpha}=\alpha \cdot \max \left(\boldsymbol{f}_{u}\right)-\boldsymbol{f}_{u} \\
\operatorname{win}^{\beta}=\mathrm{e}^{-\beta \frac{\omega(N \cdot d t)}{2 Q},}
\end{gathered}
$$

where $\boldsymbol{f}_{u}$ in Equation (11) is the amplitude spectrum of the uncompensated seismic record. The $N$ and $\mathrm{d} t$ in Equation (12) denote the sampling numbers and sampling interval. Frequency $\omega$ is the angular frequency. The quality factor $\mathrm{Q}$ can be flexibly assigned as the maximum of the input $\mathrm{Q}$ model. Parameters $a \in(1,+\infty)$ and $\beta \in[0,1]$ are two parameters that control the shape of window win $^{\mathrm{S}}$.

The design of this adaptive shaping operator aims at broadening the frequency bands more evenly in the compensation. Shaping window win $^{a}$ has the opposite trend with amplitude spectrum $\boldsymbol{f}_{u}$ in that it can restrain the compensation for frequency components that originally had a higher amplitude and promote relative compensation for low-energy components. Figure 2a uses a simulated spectrum of the Hanning window to show the shapes of window win $^{a}$ with different $a$. Increasing $a$ will make the numerical trend of window win $^{a}$ less contrary to that of $\boldsymbol{f}_{u}$ and result in a flatter window, according to which the impacts of spectral shaping will decrease. Shaping window win $^{\beta}$ resembles the amplitude term of the forward-Q filter and is designed to mitigate overcompensation for high-frequency energy by increasing the value of parameter $\beta$, as demonstrated in Figure $2 b$. The combination of shaping windows $\widehat{\boldsymbol{w i n}^{\alpha}}$ and $\boldsymbol{w i n}^{\beta}$ as a shaping operator has the potential to shape and balance the frequency spectra of the compensated records.

\subsection{Numerical Implementation}

In our implementation, shaping operator $S$ is a real diagonal matrix and is thus symmetric positive definite. This facilitates the use of some efficient algorithms, such as the conjugate-gradient method, to solve the optimization problem. Specifically, the operator can be symmetrized in the form $S=H^{\top}$ by using an invertible matrix $\boldsymbol{H}$, which can easily be obtained by taking the square root of each nonzero element in S. Equation (8) can then be expressed as

$$
\boldsymbol{U}=\boldsymbol{H}\left[\mu^{2} \boldsymbol{I}+\boldsymbol{H}^{\top}\left(\boldsymbol{A}^{\top} \boldsymbol{A}-\mu^{2} \boldsymbol{I}\right) \boldsymbol{H}\right]^{-1} \boldsymbol{H}^{\top} \boldsymbol{A}^{\top} \boldsymbol{u}
$$

(Fomel, 2007). We solved this shaping-regularization problem by using the algorithm given by Fomel (2007), which combines Equa-
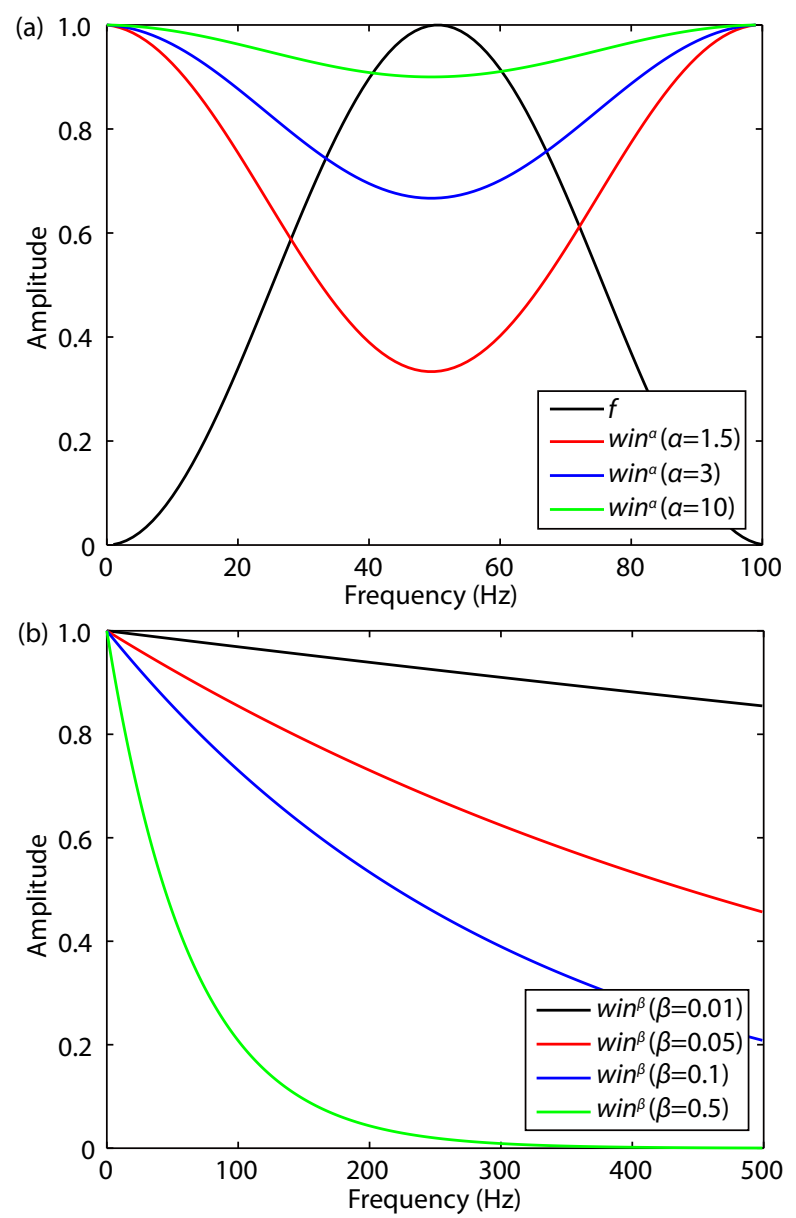

Figure 2. Relations between (a) parameter $a$ and shapes of the shaping window $\boldsymbol{w i n}^{a}$, and (b) parameter $\beta$ and shapes of the shaping window $\boldsymbol{w i n}^{\beta}$. A simulated amplitude spectrum using the Hanning window $f$ is plotted in (a) with a black line.

tion (13) and the standard conjugate-gradient iterative inversion (Hestenes and Steifel, 1952).

The numerical implementation of the proposed SSR compensation is described in pseudocode (Table1). We also briefly summarize it as follows: In Step 1, preliminary computations that obtain the incident angles of each layer $\theta_{i}$ are required to construct the offset-related $\mathrm{Q}$ filters (Equation (6)). This computation can be realized efficiently by using the ray-tracing technique, given the offset $\boldsymbol{x}$. In addition, an effective $\mathrm{Q}$ will be calculated by using $\cos \theta_{i}$ (Equation (A7)). In Step 2, we construct the offset-related forward $\mathrm{Q}$ filtering operator $\boldsymbol{A}$, with each element determined by using Equation (6). In Step 3, the shaping operator $S$ will be constructed adaptively in reference to the amplitude spectra of the data by using Equations (9) to (12). In Step 4, given the forward operator $\boldsymbol{A}$, shaping operator $\boldsymbol{S}$, and data $\boldsymbol{u}$, a conjugate-gradient iteration will be implemented to achieve the inversion-based compensation.

Two pairs of parameters, scaling factor $\mu$ and tolerance tol in the shaping regularization and $a, \beta$ that determines the shaping window, need to be adjusted in our implementation. Scaling factor $\mu$ controls the shape of operator $\boldsymbol{S}$, and larger values of $\mu$ will enforce more intense shaping on the solution. Tolerance tol is re- 
lated to the signal-to-noise (SNR) ratio of the seismic records. It can be assigned close to the noise standard deviation to avoid en-

Table 1. Pseudo code of the spectral-shaping-regularized (SSR) compensation.

Input: a. Data preparation: $u$ : single-trace seismic data; $\mathrm{d} t$ : sampling interval; $x$ : offset

b. Input velocity and $\mathbf{Q}: \boldsymbol{v}$ : interval velocity; $\mathbf{Q}$ : interval $\mathrm{Q}$ c. Parameter settings: $\mu$, tol: scaling factor and tolerance for the shaping regularization $a, \beta$ : parameters used to design spectralshaping operator $\boldsymbol{S}$

Step 1. Use the ray-tracing technique to compute the incident angle of each layer $\theta_{i}$, given the offset $x$. Then obtain the effective quality factor $Q_{e, n}$ by using $\cos \theta_{i}$ (Equation (A7)).

Step 2. Construct the offset-related forward-Q filtering operator $\boldsymbol{A}$ by using Equation (6).

Step 3. Design the shaping operator $S$ by using Equations (9) to (12) and the spectrum $\boldsymbol{f}_{u}=|\mathrm{FFT}(\boldsymbol{u})|$.

Step 4. Perform a conjugate-gradient iterative inversion (see Fomel, 2007) by using matrices $\boldsymbol{A}$ and $\boldsymbol{S}$ and input data $\boldsymbol{u}$. The inversion results will be the spectrum of the compensated data $U$.

Output: Compensated data in time domain $\boldsymbol{u}_{c}=\operatorname{IFFT}(\boldsymbol{U})$.

Note. $\operatorname{FFT}(\cdot)$ and IFFT $(\cdot)$ denote Fourier transform and inverse Fourier transform, respectively. $|\cdot|$ denotes absolute values.

hancing high-frequency artifacts in the compensation. As demonstrated in Figure 2, parameters $a \in(1,+\infty)$ and $\beta \in[0,1]$ directly influence the shapes of the window win $^{5}$ and further control the shape of the estimated model (in this case, the spectra of the compensated data). In this study, we determined the values for these two parameters through trial and error, and we found that the shaping performance would remain stable when $a$ was set close to 1 .

\section{Numerical Examples}

In these examples, we utilized common-middle-point (CMP) records synthesized in a one-dimensional layered model to test the performance of the SSR compensation. The black curves in Figure $3 a$ are the models of velocity and the quality factor. The Butterworth wavelet, as shown in Figure $3 \mathrm{~b}$ with its frequency spectrum, was used for the simulation. The zero-offset travel time at each interface was calculated based on the velocity model, where the reflection coefficients were assigned as 1 . The records were then constructed through convolving reflection coefficients with the wavelet, as shown in Figure 4a. Twenty identical traces were synthesized, with the interval set as $200 \mathrm{~m}$. Each trace included 2,000 samples, and $0.002 \mathrm{~s}$ was the sampling interval.

The attenuated records in Figure $4 \mathrm{~b}$ were obtained by offset-related full forward-Q filtering, with the accurate velocity and $Q$ models shown in Figure 3a as inputs. Figure 4c shows representative traces of the records in Figure 4b. The impacts of seismic ray paths on the offsets were considered, resulting in the variation in attenuation across traces. The proposed SSR method was used to compensate for the attenuated records. In this scenario with a noise-free signal and accurate velocity and $\mathrm{Q}$ models, the shaping operator was designed to be an identity matrix that would turn the inversion-based compensation into an unregularized one. The

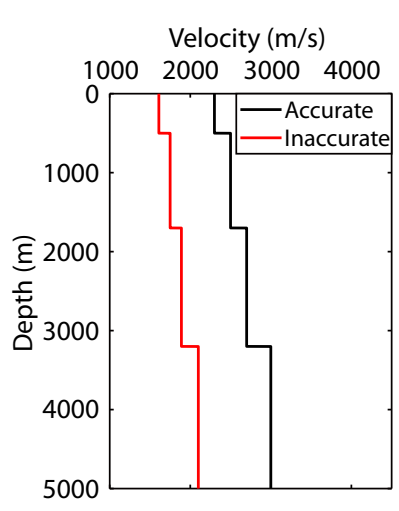

(a)
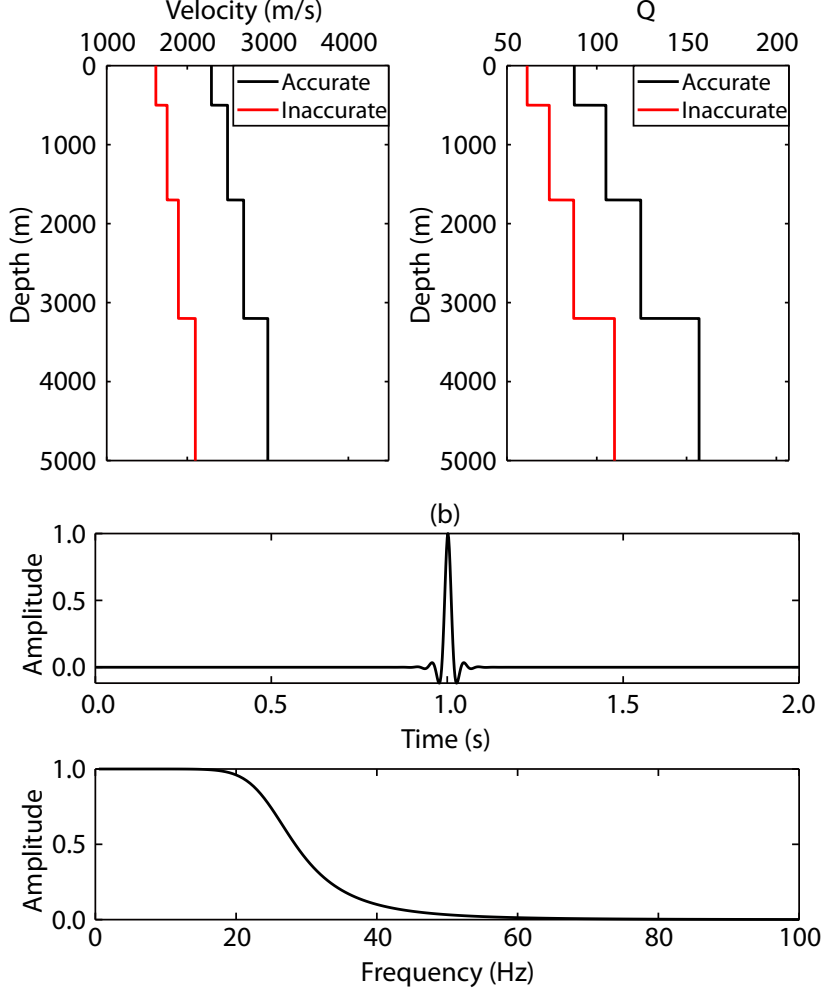

Figure 3. (a) Velocity and $Q$ models. Black curves denote accurate models, and red curves denote inaccurate estimates. (b) The Butterworth wavelet and its frequency spectrum.

compensated trace at the farthest offset was plotted against the original trace at the bottom of Figure 4c. The consistency between the two signals verified the feasibility of the method.

To be more consistent with the field cases, we then implemented more complex processing flows. Wavelets in the attenuated records were first relocated according to the travel time calculated by ray tracing. Random noise was then added to the records, as shown in Figure $5 \mathrm{a}$. The SNR of the records was $7 \mathrm{~dB}$ and was computed by SNR $=\frac{\left\|X^{*}\right\|}{\left\|X-X^{*}\right\|}$, where $\boldsymbol{X}$ and $\boldsymbol{X}^{*}$ denote noisy data and the noise-free model, respectively. Note that we did not design an extremely noisy example because we hoped to evaluate the spectral-shaping performance of the designed operator. Another synthetic example with strong noise contamination is given in the Discussion section to demonstrate the robustness of this method. A normal moveout (NMO) correction was implemented, and the corrected records are shown in Figure $5 \mathrm{~b}$. This figure explicitly shows that the NMO correction stretched the waveforms at the far offsets, which could directly influence the compensation performance. Therefore, a wavelet stretching correction was further applied on the records, in which the wavelets in the first trace were regarded as nonstretched and used to obtain the stretching factor for the spectral scaling of the other traces (Xu $Y$ and Chopra, 2007). The stretching-corrected records are shown in Figure 5c, and a seismic attenuation compensation was implemented on the records by using inaccurate velocity and $\mathrm{Q}$ models, as shown by the red lines in Figure 5 a. 

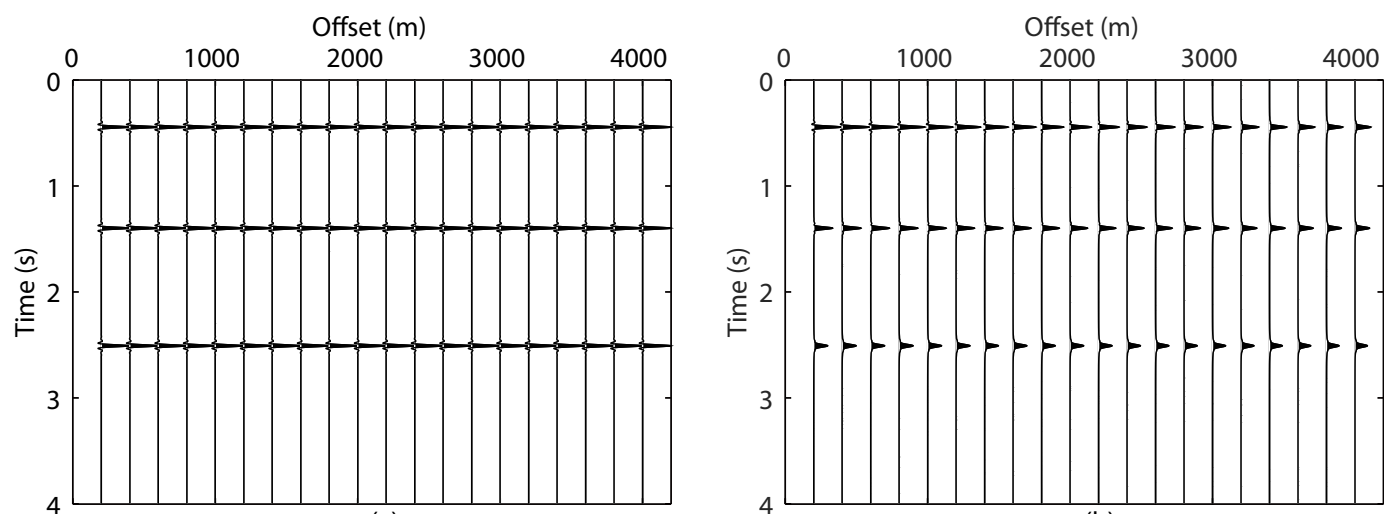

(a)

(b)

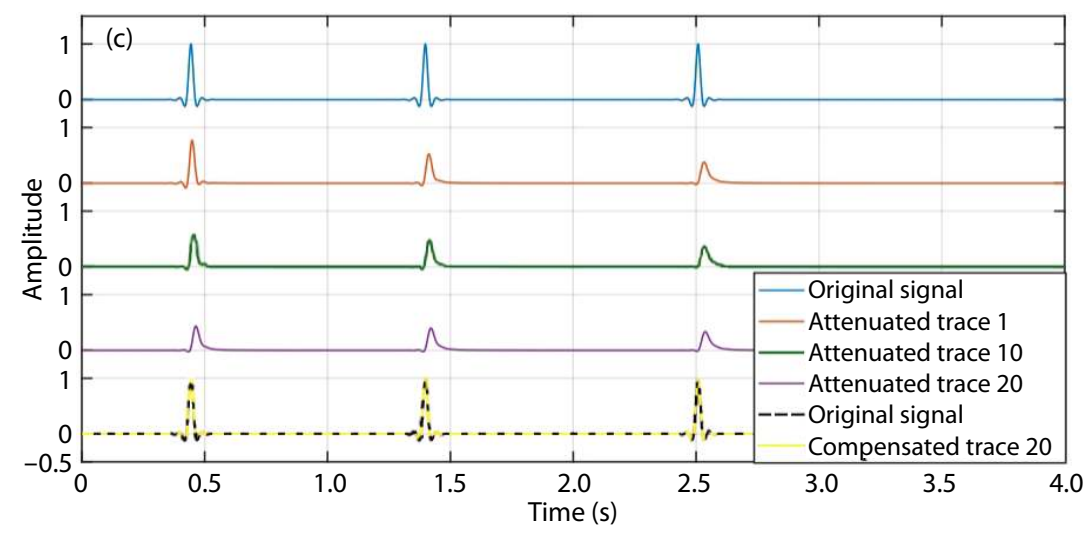

Figure 4. (a) Original records composed of 20 identical traces. (b) Records attenuated by the offset-related full forward-Q filter. (c) Single traces of the attenuated records in (a) and compensation of the farthest-offset trace by using the offset-related inverse- $Q$ filter.

Stabilized inverse-Q filtering (Wang YH, 2002) was used as a standard approach to make comparisons with the SSR compensation. The compensated records using this standard method are plotted in Figure $5 d$, with the stabilized factor set as 0.06 . Figures $5 e$ and $5 f$ were computed by using the SSR compensation, in which the scaling factors were 80 and 40 , respectively. The tolerance was set as $10^{-4}$. Because of the inaccuracy of the $Q$ and velocity models, the wavelet phases would differ before and after phase correction, which would influence our comparisons and analysis. Fortunately, phase compensation has been proved to be unconditionally stable (Wang YH, 2002). In addition, the amplitude loss and velocity dispersion were decoupled in the $\mathrm{Q}$ filters (Equations (4) to (6)), and the amplitude was available to be solely compensated in the proposed compensation scheme. Hence, amplitude-only $\mathrm{Q}$ filtering was applied in this synthetic case. Influenced by the instability of the wavelet stretching correction, shallow records at far offsets were not well recovered. Except for the distorted samples, the records in Figures $5 d$ and $5 f$ were both well compensated without significant noise enhancement, indicating the performance of the proposed compensation scheme was competitive. Because of the stronger scaling (larger $\mu$ ), the compensation in Figure 5e appeared to be inadequate for deeper wavelets at larger offsets, as indicated by the red arrow.

The frequency spectra of wavelets in the records of Figures $5 c$ to $5 \mathrm{f}$ were analyzed. The records were windowed in $0-1,000 \mathrm{~ms}, 1$, $000-2,000 \mathrm{~ms}$, and 2,000-3,000 $\mathrm{ms}$, and the spectra were computed separately within these windows, as plotted in Figures $6 a$,
$6 \mathrm{~b}$, and $6 \mathrm{c}$ in different colors. The adaptive shaping windows win $^{5}$, $\boldsymbol{w i n}^{\alpha}$, and $\boldsymbol{w i n}^{\beta}$, which were used to construct the shaping operator, are plotted in Figure $6 \mathrm{~d}$ in different colors, and $a$ and $\beta$ were assigned to be 1.3 and 0.01 , respectively. A relatively small value was assigned to $a$ to amplify the spectral-balancing function of window win $^{a}$. Because the noise level was low in this case, we assigned an extremely small value to $\beta$, which made window win $^{\beta}$ almost ineffective in the high-frequency attenuation. In contrast with the spectra of the compensated records in Figures $5 d$ and $5 f$, the low-frequency components were better preserved for the wavelets in Figure 5e, whereas the performance of the high-frequency compensation was still competitive with the other two cases. The performance of the low-frequency preservation became more prominent for deeper wavelets (indicated by the dashed red ellipses), although their amplitudes may not have been fully compensated because of the strong scaling. Stabilized inverse- $Q$ filtering, on the other hand, was able to adjust the energy distribution of the frequency spectra through the stabilized factor, whereas it improved the high-frequency compensation at the cost of low-frequency energy, and vice versa.

\section{Field Data Examples}

The SSR compensation was applied on common-reflection-point (CRP) gathers, which were obtained through the workflow of prestack time migration (Yilmaz, 2001). In total, 1,900 gathers were tested, and each gather consisted of 60 traces. Records from 2 to $4 \mathrm{~s}$ were used, and the sampling interval was $0.002 \mathrm{~s}$. In each 

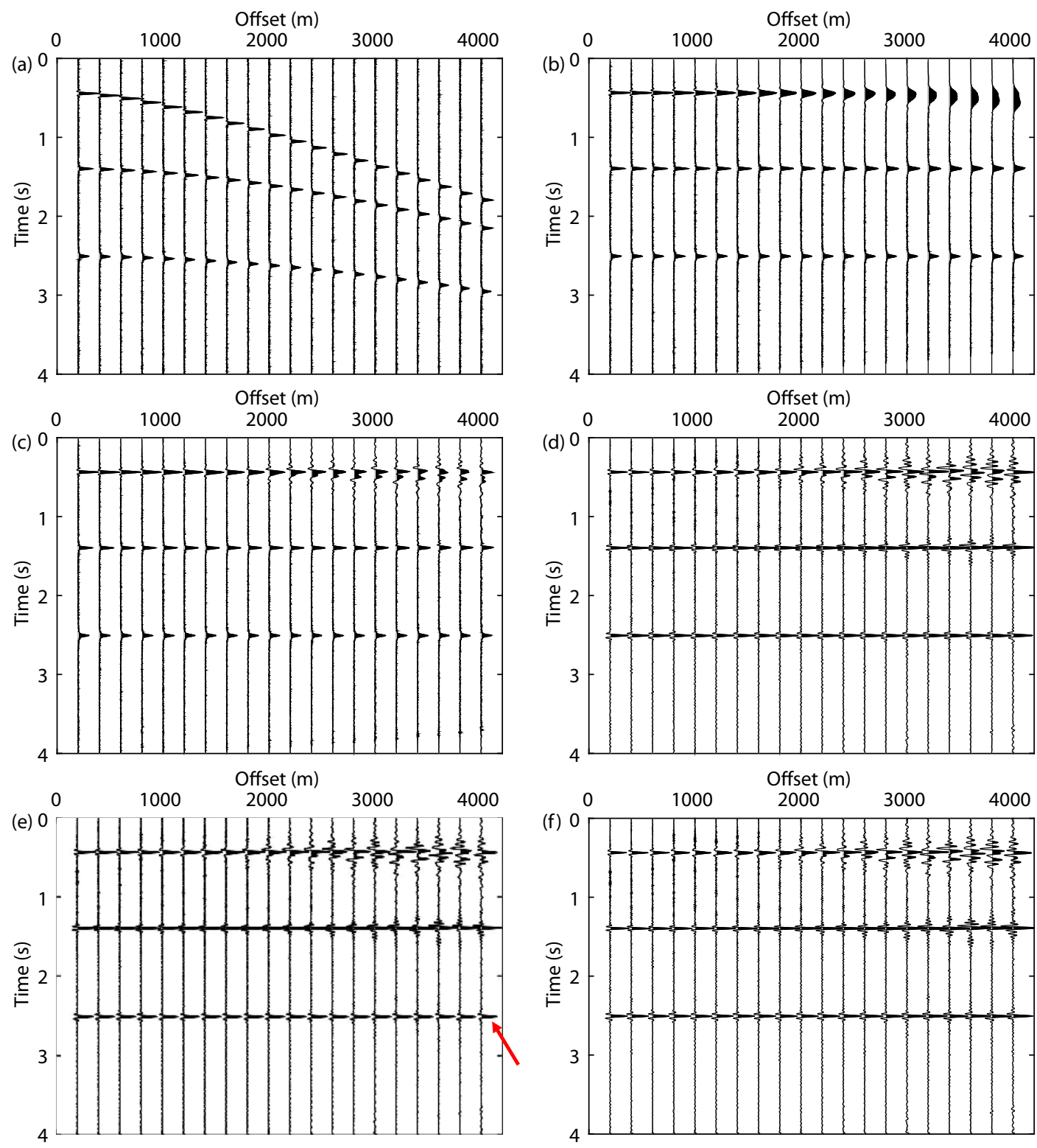

Figure 5. (a) Attenuated records contaminated by noise. (b) Normal moveout-corrected records. (c) Stretching-corrected records. Records compensated by using (d) stabilized inverse- $Q$ filtering, (e) spectral-shaping-regularized (SSR) compensation with a scaling factor of $\mu=80$, and (f) SSR compensation with a scaling factor of $\mu=40$. Inaccurate velocity and Q models in Figure $3 a$ (red curve) were used for compensation. The red arrows in (e) denotes the position where the wavelet was not fully compensated.

gather, the minimum and maximum offsets were, respectively, $250 \mathrm{~m}$ and $6,150 \mathrm{~m}$, with an interval of $100 \mathrm{~m}$. Figure 7a shows one of the CRP gathers, and the corresponding velocity and $\mathrm{Q}$ models of this gather are plotted in Figure 8 . The interval velocity model in Figure 8 was transformed from the root-mean-square velocity by using the Dix equation (Dix, 1955). The interval $Q$ was then calculated based on the empirical $\boldsymbol{V}_{p}-Q$ relation proposed by Li QZ $(1993,2017)$, which describes a general trend of Q distribution varying with P-wave velocity. Before compensation, CRP gathers were processed by an NMO stretching correction, and the corrected gather is shown in Figure 7b. A summation of the first 20 traces in each gather was used to scale the far-offset data ( $X u Y$ and Chopra, 2007).
Stabilized inverse-Q filtering and SSR compensation were implemented on the stretching-corrected gathers. Similar to the strategy in the second synthetic case, only amplitude compensation was performed on the records because the phase corrections would lead to dislocated events before and after compensation, making the comparisons less intuitive. In addition, the phase corrections are unconditionally stable and do not add complexity to our comparisons. Factor $\sigma$ was set as 0.03 in the stabilized inverseQ filtering, whereas the scaling factor was set as 20 in the SSR compensation. The CRP gathers were nearly noise free, and the tolerance tol was assigned as $10^{-9}$. Parameters $a$ and $\beta$ for the adaptive shaping operator were 1.3 and 0.1 . Figure $7 c$ and $7 d$ show the compensated results using these two methods. 

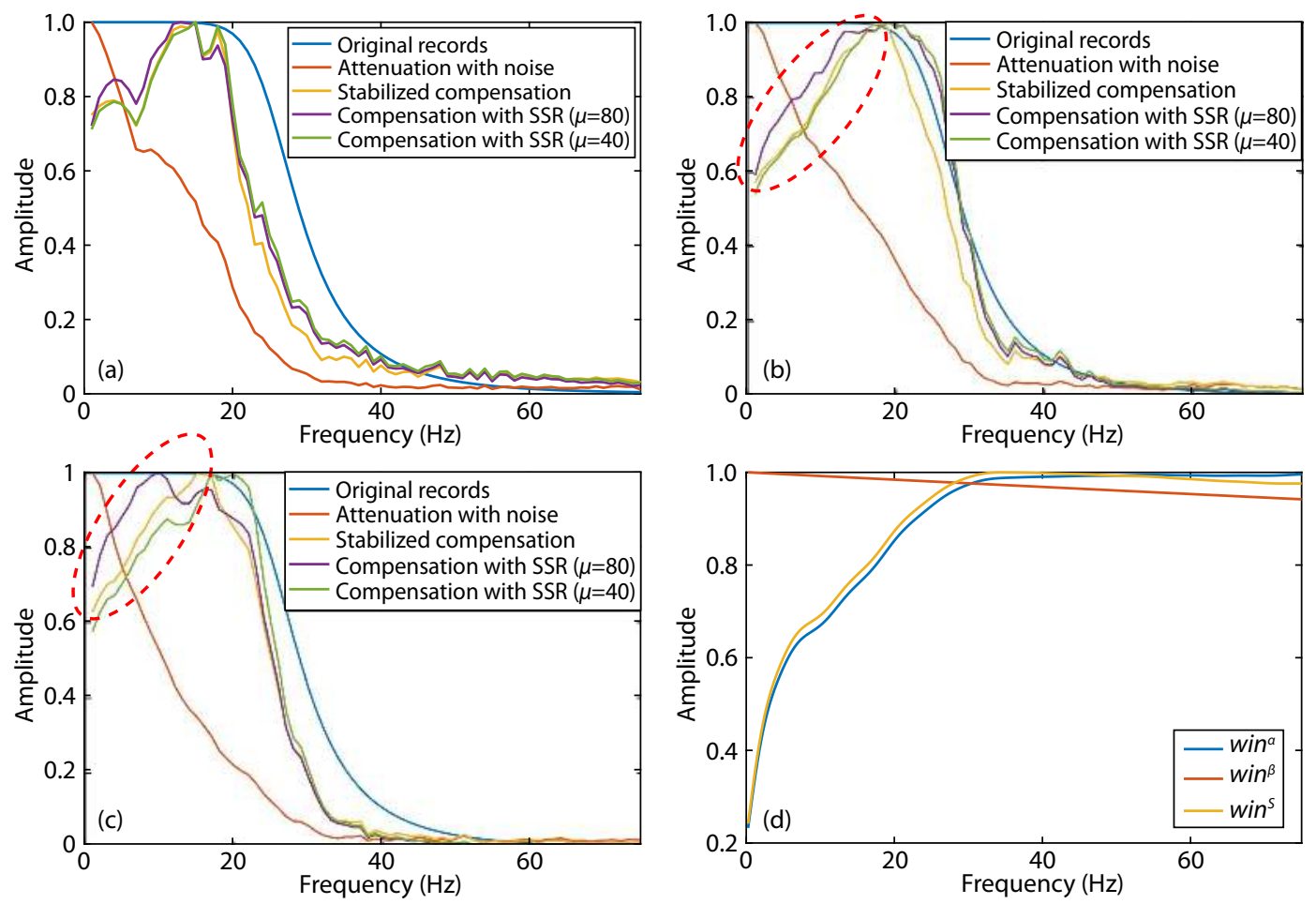

Figure 6. Normalized amplitude spectra of wavelets in the synthetic records of Figure $5 c, 5 d, 5 e$, and $5 f$ within time windows (a) $0-1,000$ ms, (b) 1,000-2,000 ms, and (c) 2,000-3,000 ms. (d) Shaping windows designed for the synthetic case. The dashed ellipses in (b) and (c) highlight the preserved low-frequency amplitude after compensation.

To further compare the results of compensation, partial-offset stacking was applied on the CRP gathers. Traces with offsets between 250 and 3,200 m were partially stacked, and traces with offsets greater than 3,200 m were stacked together. Partial-offset stacks of the original CRP gathers are shown in Figures 9a and 9b, whereas partially stacked profiles of the compensated CRP gathers using stabilized inverse- $Q$ filtering and SSR compensation are shown in Figures 9c, 9d, 9e, and 9f. Resolutions of the stacked profiles were enhanced after compensation by using these two methods. Compared with the results compensated by stabilized inverse-Q filtering, the partial stacks after SSR compensation showed seismic events with more enriched low-frequency information. In the near-offset stacks, these improvements were prominent at the locations marked with arrows in Figures $9 \mathrm{c}$ and 9e. For the far-offset profiles in Figures $9 d$ and $9 f$, the difference can clearly be observed in records after $3 \mathrm{~s}$, where seismic events processed by SSR compensation are more continuous. Local windows of the partial-offset stacks, as indicated by the colored boxes in Figure 9, are shown in Figure 10. In these magnified windows, high-frequency artifacts, as denoted by the arrows, are more visibly exposed in the profiles compensated by stabilized inverse-Q filtering, especially in Figures $10 \mathrm{~h}$ and $10 \mathrm{k}$. The local stacks processed by SSR compensation showed relatively stable results.

Finally, frequency spectra of the seismic data before and after compensation were analyzed. Shaping windows $\boldsymbol{w i n}^{S}, \boldsymbol{w i n}^{a}$, and $\boldsymbol{w i n}^{\beta}$ for this field example are plotted in Figure 11a. Frequency spectra of the CRP gathers in Figures $7 a, 7 c$, and $7 d$ are shown in Figure $11 \mathrm{~b}$. Compared with the original spectrum, the spectra of the compensated gathers using the two methods both had com- pelling high-frequency enhancement, whereas the low-frequency energy of the compensated spectrum using stabilized inverse-Q filtering showed relative decays. In contrast, the SSR compensation performed better in preserving the low-frequency energy, as shown by the red dashed ellipse in Figure $11 \mathrm{~b}$. This result indicates that the spectral-shaping performance of the shaping operator was competitive. The spectra of the partial-offset stacks are shown in Figures 11c and 11d. Again, the spectral bandwidth of the profiles was wider and the low-frequency energy was better preserved after being processed by the SSR compensation. The red dashed ellipses in these two figures delineate the preserved low-frequency energy. Compared with the spectrum of the far-offset profile compensated by stabilized inverse-Q filtering, the highfrequency energy was slightly undercompensated for the result obtained by the SSR compensation. This under-compensation resulted from the magnitude of scaling factor $\mu$ and could be mitigated by reducing its value. We further used a smaller scaling factor $(\mu=5)$ to compensate for the CRP gathers. The corresponding spectra of partial stacks are also plotted in Figures 11c and 11d, which show that the energy around $40 \mathrm{~Hz}$ was better compensated than that with a larger scaling factor $(\mu=20)$. On the other hand, the ability to maintain the low-frequency energy became weaker because of the smaller scaling factor.

\section{Discussion}

In this section, we first demonstrate the influence of inaccurate velocity and $\mathrm{Q}$ models on the performance of the attenuation compensation. For the $\mathrm{Q}$ filters used in this study (Equations (3) to (6)), alteration of the $Q$ models would explicitly change the mag- 

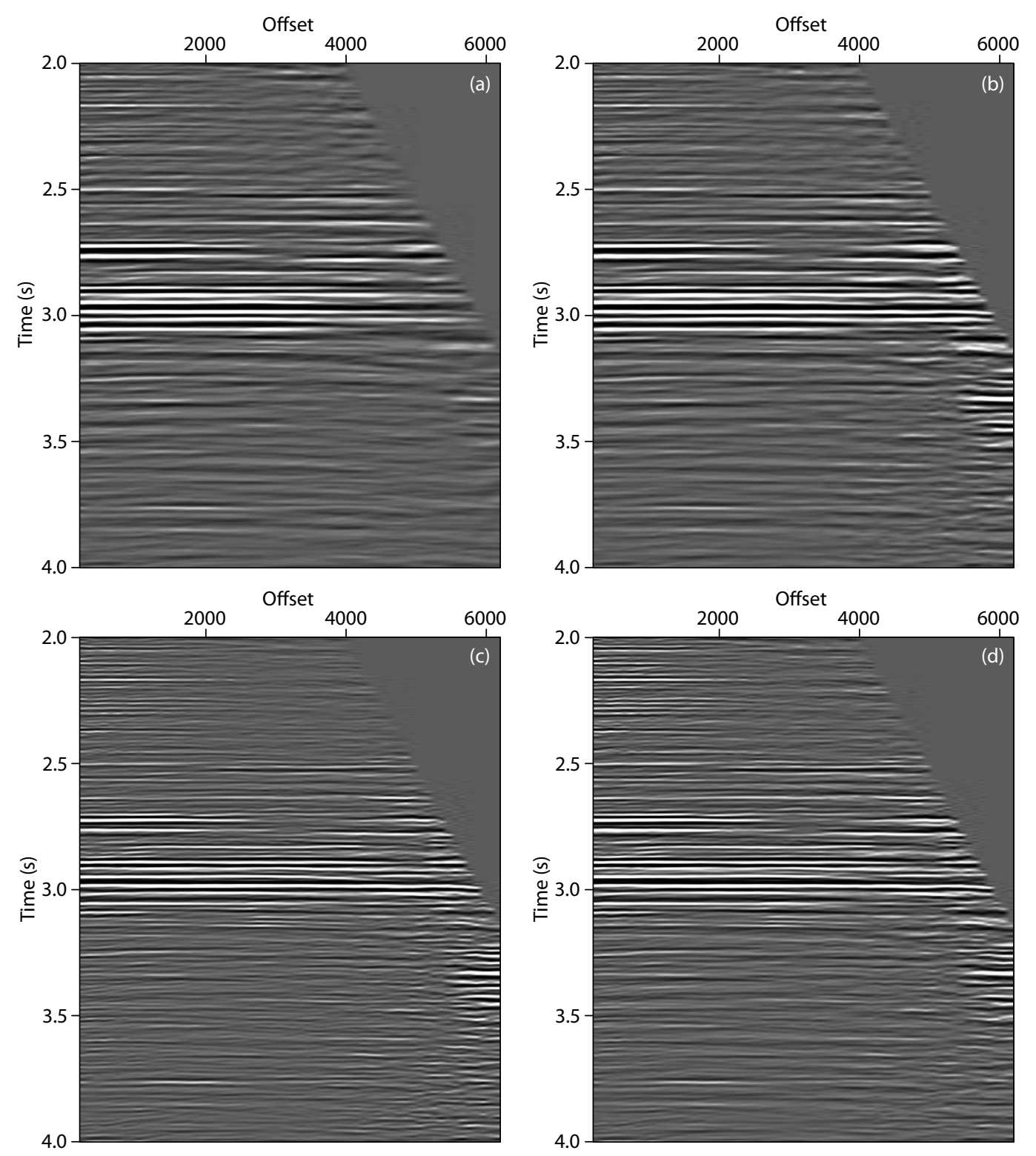

Figure 7. (a) Original common-reflection-point gather. (b) Stretching-corrected gather. Gathers compensated by using (c) stabilized inverse-Q filtering and (d) spectral-shaping-regularized compensation.

nitude of the $Q$ filters, whereas the velocity models would influence the filters by changing the incident angles $\theta$. We used the maximum-offset trace in Figure $4 \mathrm{c}$ to analyze the impacts of biased velocity and $Q$ values on the compensated wavelets. Figures $12 \mathrm{a}$ and $12 \mathrm{~b}$ compare the waveforms of the deepest wavelet in this trace after compensation by different scaling of the accurate velocity and $\mathrm{Q}$ models. We could observe that a lower velocity and $\mathrm{Q}$ would overcompensate for the seismic attenuation. Compared with the original wavelet, the compensated wavelets were increased in amplitude and delayed in phase. On the other hand, velocity and $\mathrm{Q}$ models larger than the accurate ones could lead to under-compensation. The frequency spectra of the compensated wavelets were similarly affected by the inaccurate velocity and $\mathrm{Q}$ models. Figures $12 \mathrm{c}$ and $12 \mathrm{~d}$ show that the spectra of compensated wavelets was overboosted with lower velocity and $Q$ values, whereas larger input models failed to com- pensate completely for the spectral energy. The ordinate magnitudes of the waveforms and the spectra in Figure 12 indicate that the stability of compensation was more sensitive to the accuracy of the $Q$ models than the velocity models.

Estimating interval velocity and $\mathrm{Q}$ models can be difficult in field applications. As mentioned in the field data examples, we obtained the interval velocity and $Q$ models used for compensation in an indirect way, which could be regarded as reasonable according to the results. Nevertheless, reliable estimates of (interval) Q and velocity are still the priority for an accurate and stable compensating process. The recent increase in research focusing on the wave-equation inversion method, such as the multi-parameter full-wave inversion (Kamei and Pratt, 2013; Bai T et al., 2017), provides unprecedented prospects for more precise velocity and $Q$ estimations. 

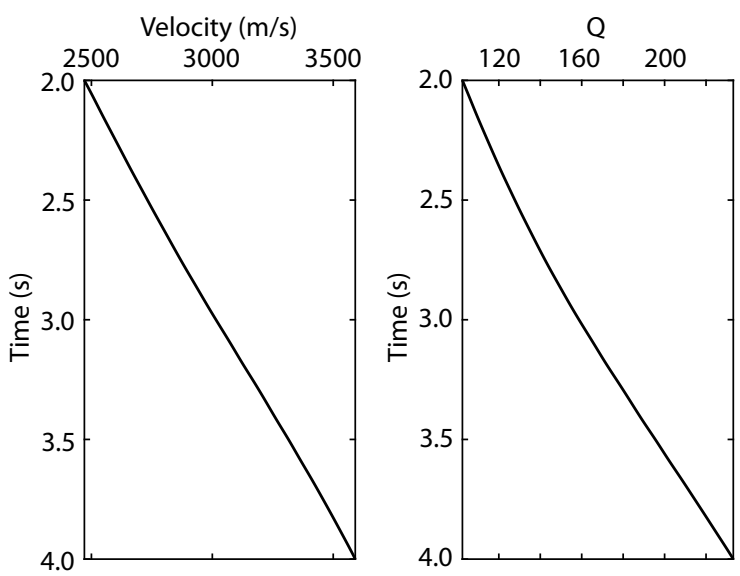

Figure 8. Velocity and Q models for the seismic gathers in Figure 7.
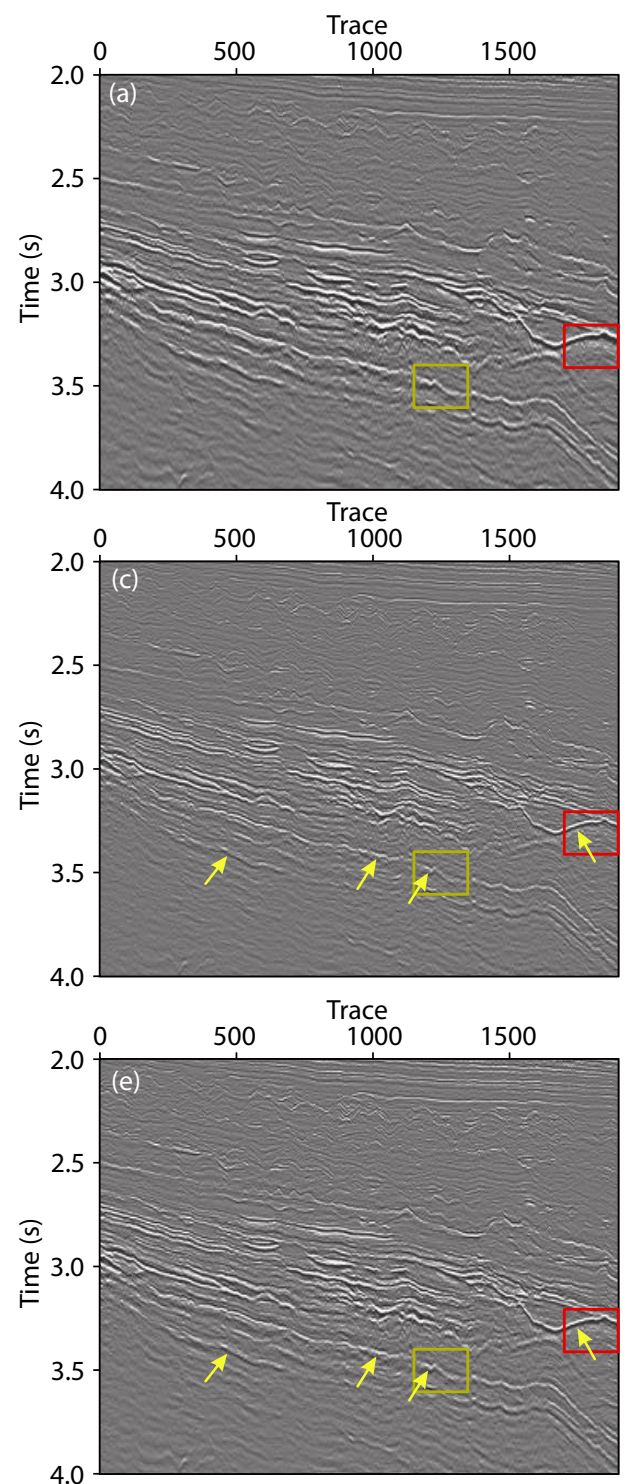

We further analyzed the robustness of the SSR compensation. In our computational scheme, the scaling factor $\mu$ mainly performed spectral shaping on the compensated records. Tolerance tol played a more critical role in rejecting the noise in the compensation. As shown in Figure 13a, high-intensity random noise was added to submerge the seismic events ( $S N R=-3.3 \mathrm{~dB}$ ). An NMO correction and a stretching correction were then applied on the noisy records, as shown in Figures 13b and 13c. We used the SSR compensation to process records with different tolerances tol, and the scaling factor $\mu$ was kept constant at 10 . The compensated records are shown in Figures 13d to 13f. With the increase in tol, the noise was gradually suppressed, which contributed to a more stable compensation. On the other hand, larger tol values could restrain the compensation to different extents. Hence, the parameter acted as a numerical tradeoff between noise suppression
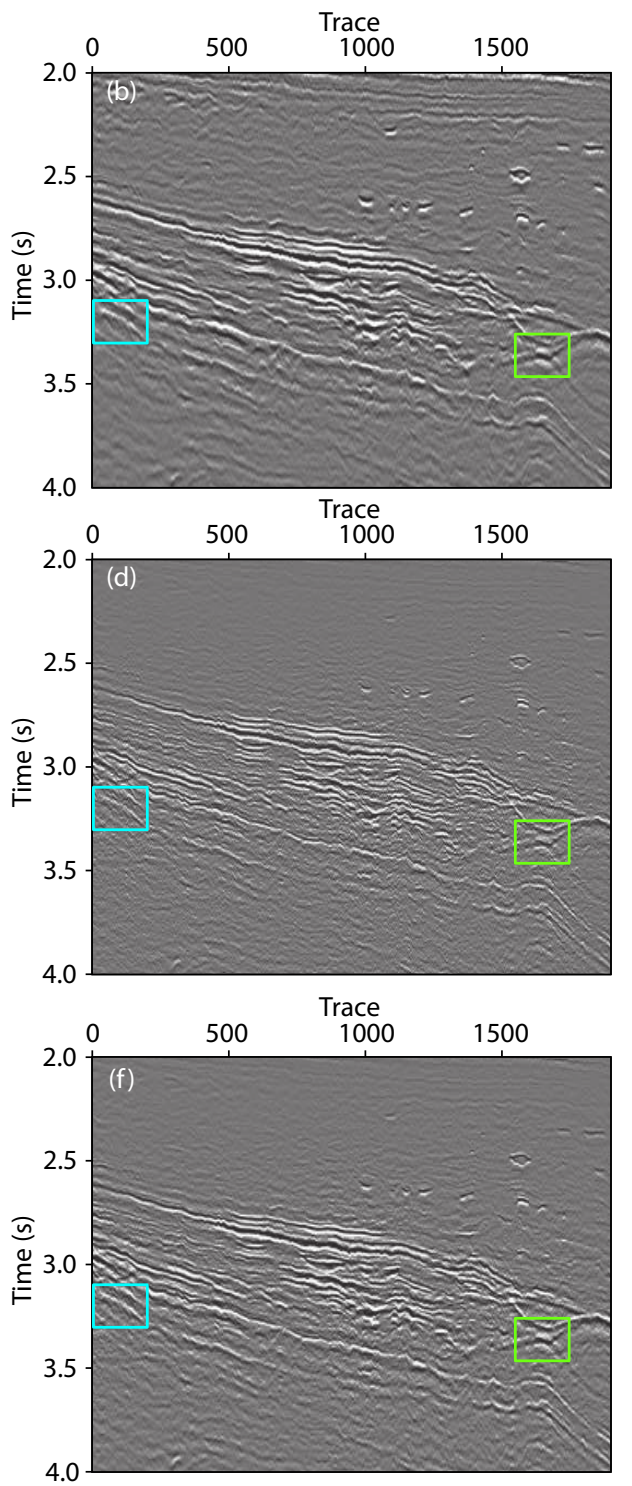

Figure 9. Partial-offset stacking of ( $a, c, e)$ near offsets between 250 and 3,200 m and (b, d, f) far offsets between 3,200 and 6,150 m, respectively, for the original gathers $(a, b)$, compensated gathers using stabilized inverse- $Q$ filtering $(c, d)$, and spectral-shaping-regularized compensation (e, f). The yellow arrows denote the positions on the gathers where the spectral-shaping-regularized compensation performed better than stabilized inverse-Q filtering in preserving low-frequency energy. The colored boxes represent windows that are further analyzed in Figure 10 

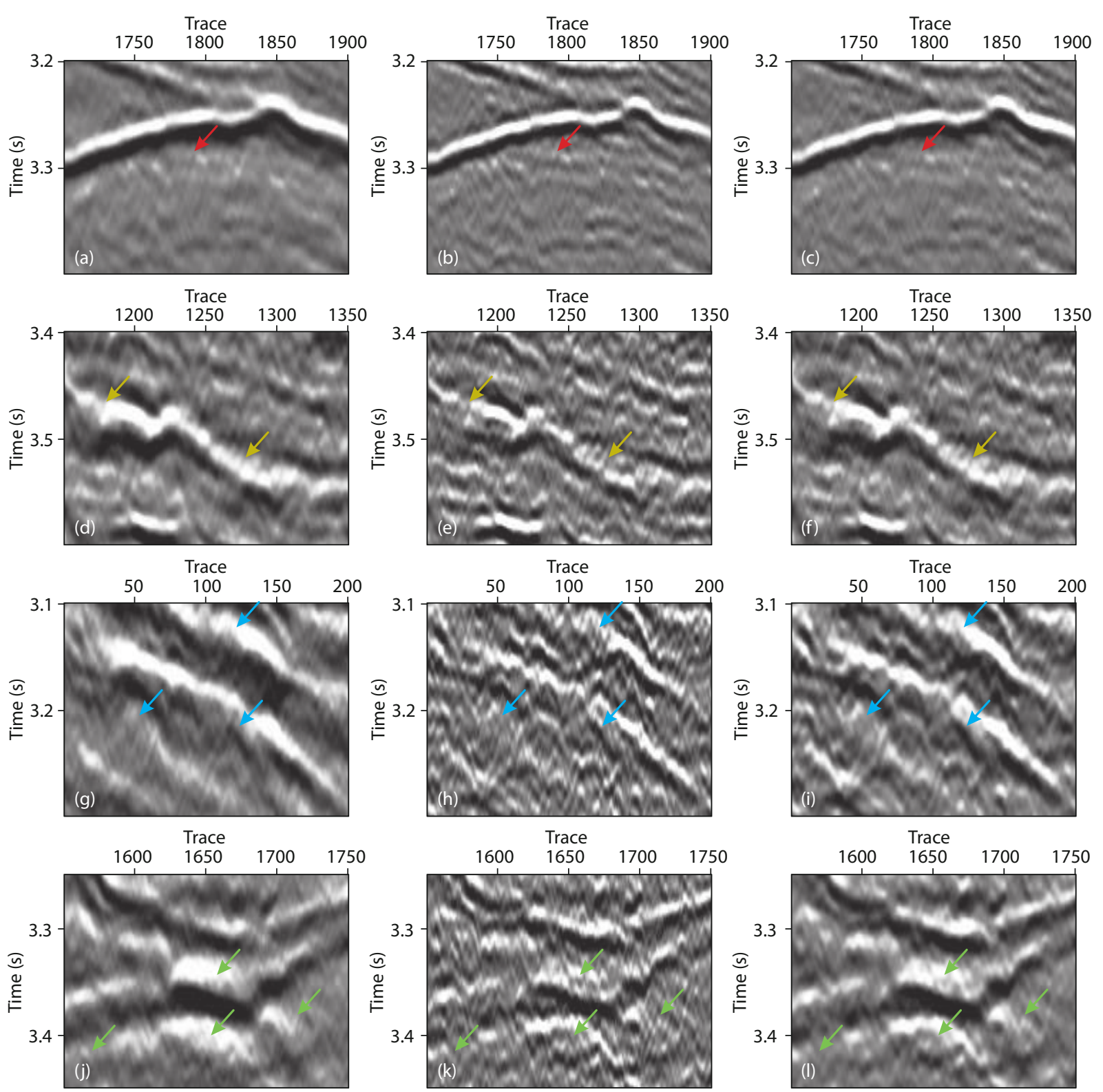

Figure 10. Locally magnified windows of the $(a-c)$ red boxes, $(d-f)$ yellow boxes, $(g-i)$ blue boxes, and (j-l) green boxes in Figure 9 . Local stacks of original gathers (left), and compensated ones with stabilized inverse-Q filtering (middle) and spectral-shaping-regularized compensation (right). The colored arrows in each panel denote the positions where the spectral-shaping-regularized compensation performed better than the stabilized inverse-Q filtering in preserving low-frequency information.

and attenuation compensation.

We also compared the computational costs between the SSR compensation and the stabilized inverse- $Q$ filtering. The efficiency of the stabilized inverse-Q filtering (Wang $\mathrm{YH}, 2002$ ) has been generally accepted. We used the noise-free synthetic example in Figure $4 \mathrm{~b}$ and the input velocity and $\mathrm{Q}$ models in Figure $3 a$ to test the computational costs of these two methods. The synthetic data included 1,000 samples and 20 traces, which were generally consistent in volume with the actual seismic records. In computing the shaping regularization, tolerance tol was set to zero so that the iterations would cease at the maximum number of iterations (20). We repeated the same computations more than 30 times and used the average of each run to quantify the computational costs. Table 2 lists the representative computation times for these two methods. Overall, the test suggests that the computational cost of the SSR compensation was twice as much as the conventional inverse-Q filtering. For the SSR compensation, we further divided the computation into three portions to analyze the cost of each portion separately. It was not surprising that the forward-Q filters and the iterative shaping regularization almost equally shared the time spent in the SSR compensation. Other computations, including ray tracing, effective $Q$ models, and FFT/IFFT, accounted for only a negligible fraction of the total computational burden.

It is also worth noting that the low frequency in our study was defined in a relative sense. A common belief is that the low-fre- 

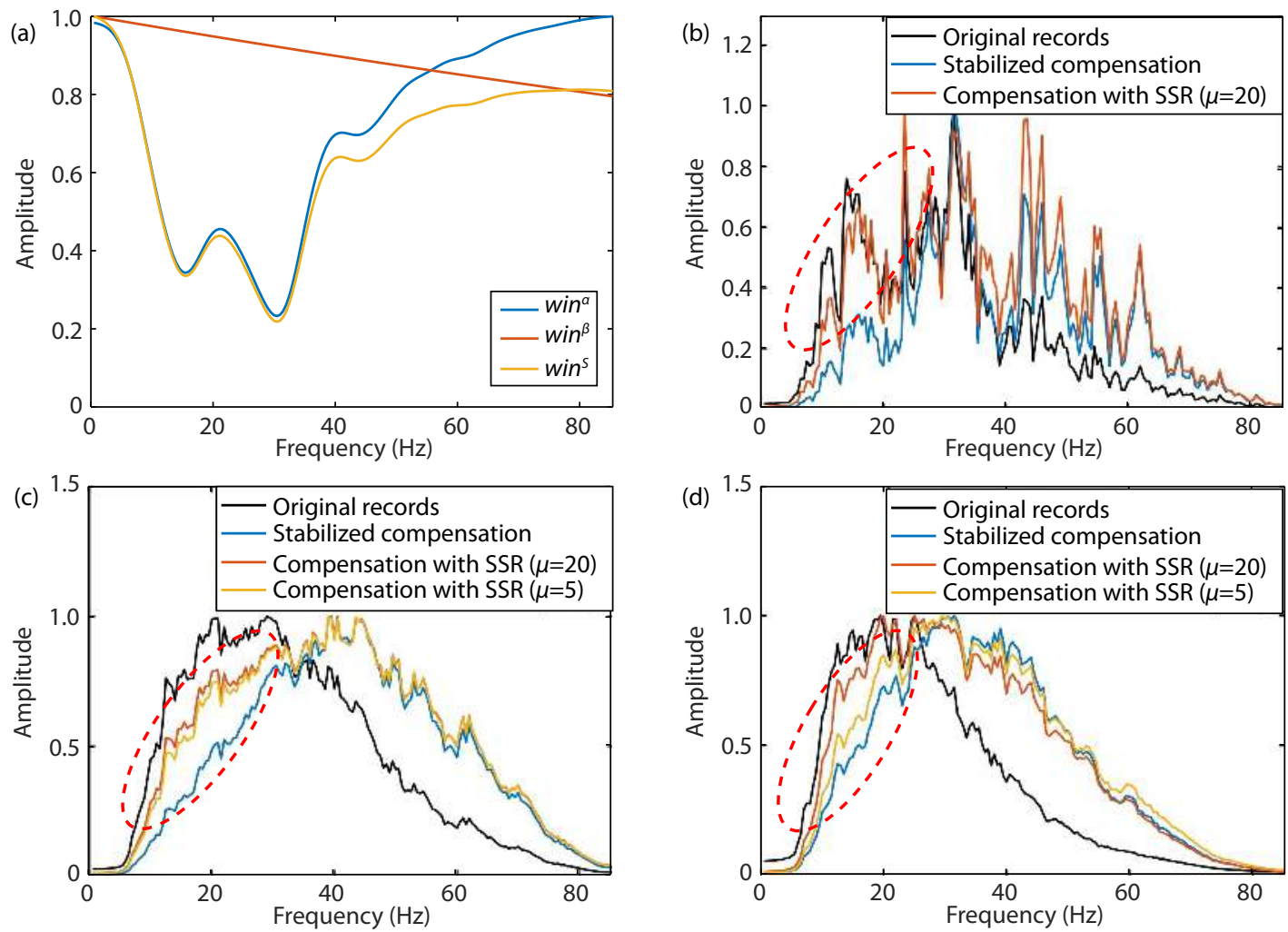

Figure 11. (a) Shaping windows designed for the field case. Shaping window win $^{a}$ is smoothed. Normalized amplitude spectra of (b) commonreflection-point gathers in Figure 7, (c) near-offset stacks, and (d) far-offset stacks. The dashed ellipses in (b) to (d) highlight the preserved lowfrequency amplitude after compensation.
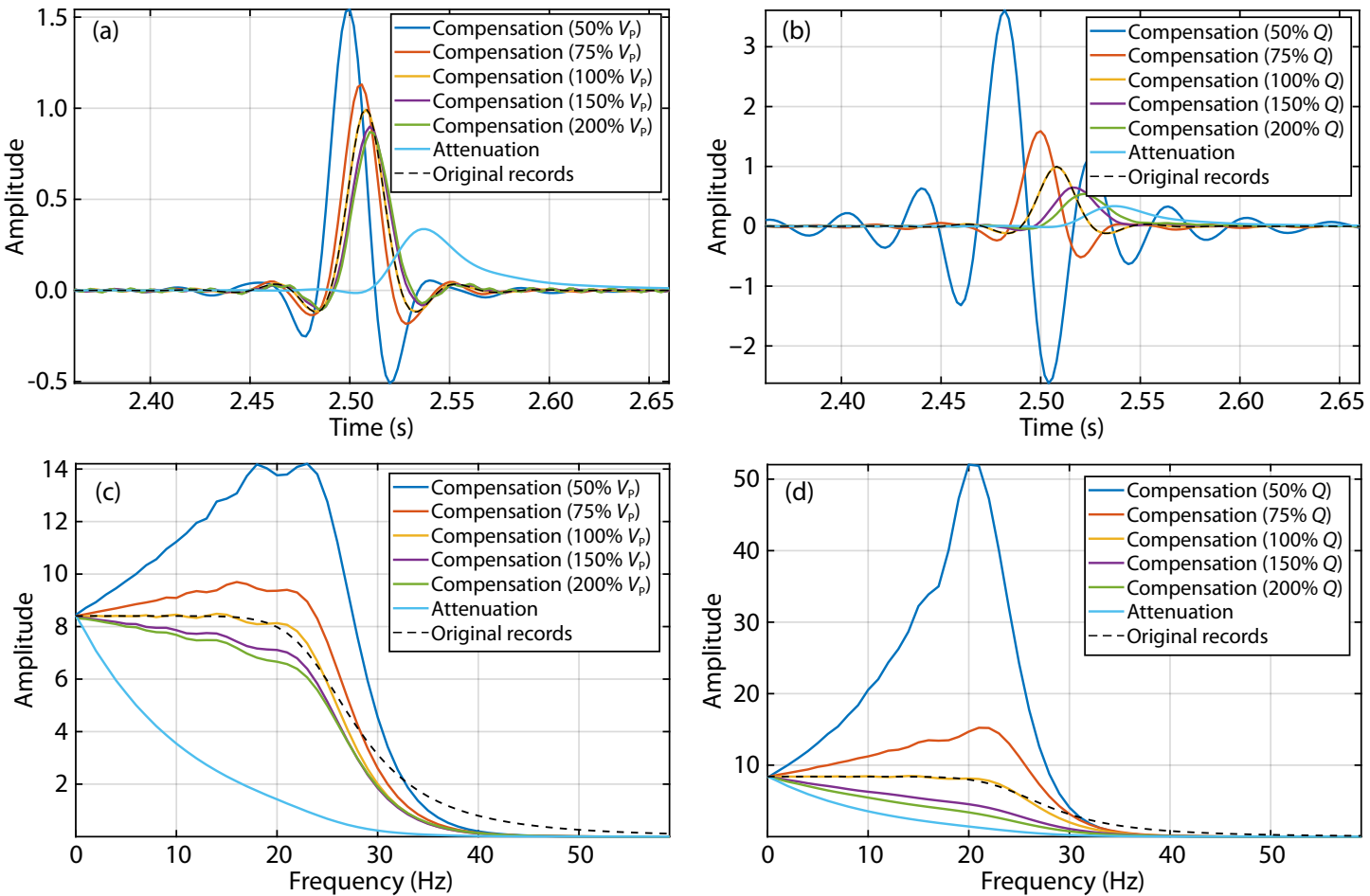

Figure 12. Wavelets compensated by spectral-shaping-regularized compensation with different (a) velocities and (b) Q. (c, d) Frequency spectra of the wavelets in (a) and (b). The deepest wavelet in the farthest-offset trace of Figure $4 c$ was used for the analysis. 

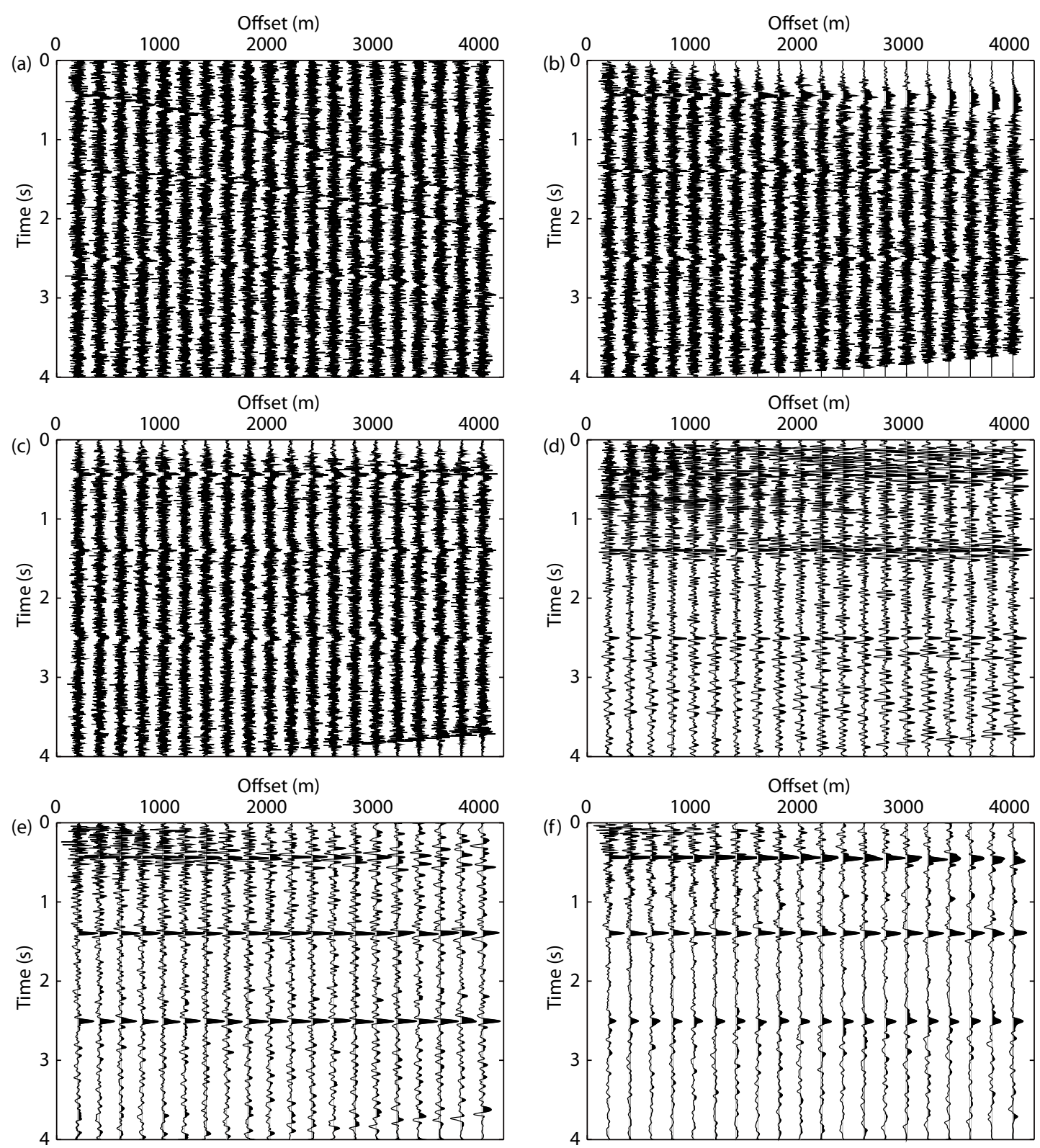

Figure 13. (a) Synthetic common-middle-point records with strong noise. (b) Normal moveout-corrected records. (c) Stretching-corrected records in which the first trace is treated as nonstretched wavelets. Records compensated by the spectral-shaping-regularized compensation with (d) $t o l=0.02$, (e) tol $=0.1$, and (f) $t o l=0.2$.

Table 2. Representative computational time of spectral-shaping-regularized (SSR) compensation and stabilized inverse-Q filtering.

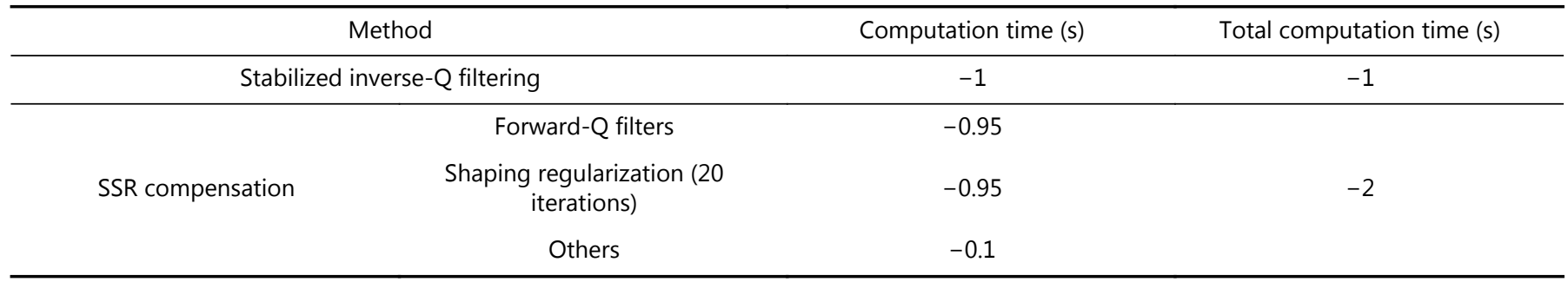

Note. The computations were carried out using MATLAB 2018a on a Lenovo standalone workstation with a 11th Generation Intel Core Processor $(3.00-4.80 \mathrm{GHz})$ and $16 \mathrm{~GB}$ of memory. 
quency range for surface seismic exploration is less than $10 \mathrm{~Hz}$. In our field example, the preserved "low-frequency" energy primarily ranged within 10 to $20 \mathrm{~Hz}$ because the energy below $10 \mathrm{~Hz}$ had totally been lost in the original records (Figure 11). Although this work focuses on preserving the existing low-frequency energy in the process of high-frequency compensation, knowing how to recover energy less than $10 \mathrm{~Hz}$ in this case would involve a study of low-frequency reconstructions (Ding $Y$ et al., 2019).

\section{Conclusions}

We designed an adaptive shaping operator based on the frequency spectra of attenuated seismic data to achieve a spectralshaping function in inversion-based attenuation compensation. The shaping operator was successfully included in the inversion via the shaping regularization. Regularized with the adaptive shaping operator, the inversion-based compensation showed better performance on low-frequency energy preservation as well as stable high-frequency compensation. The competitive performance of the SSR compensation was illustrated and verified through synthetic tests and a field data application. When limited by factors from various perspectives, real seismic attenuation can hardly be estimated accurately, nor can it easily be recovered. Enhancing the data resolution and maintaining the frequency bandwidth could be more practical goals for seismic attenuation compensation. The proposed approach therefore has considerable significance for practical applications.

\section{Acknowledgments}

This research was supported by the National Natural Science Foundation of China (No. 41930429), 14th Five-Year Prospective and Basic Research Program of the CNPC (No. 2021DJ3506), the China National "111" Foreign Experts Introduction Plan for Tight Oil \& Gas Geology and Exploration, and the Deep-Ultradeep Oil \& Gas Geophysical Exploration and Qingdao Applied Research Projects.

\section{References}

Bai, M., Chen, X. H., Wu, J., Chen, Y. K., Liu, G. C., and Wang, E. J. (2016). Multiplecomponent Gaussian beam reverse-time migration based on attenuation compensation. Chin. J. Geophys. (in Chinese), 59(9), 3379-3393. https://doi.org/10.6038/cjg20160921

Bai, T., Tsvankin, I., and Wu, X. M. (2017). Waveform inversion for attenuation estimation in anisotropic media. Geophysics, 82(4), WA83-WA93. https://doi.org/10.1190/geo2016-0596.1

Bickel, S. H., and Natarajan, R. R. (1985). Plane-wave $Q$ deconvolution. Geophysics, 50(9), 1426-1439. https://doi.org/10.1190/1.1442011

Biot, M. A. (1956). Theory of propagation of elastic waves in a fluid-saturated porous solid. II. Higher frequency range. J. Acoust. Soc. Am., 28(2), 179-191. https://doi.org/10.1121/1.1908241

Blanch, J. O., Robertsson, J. O. A., and Symes, W. W. (1995). Modeling of a constant $Q$ : methodology and algorithm for an efficient and optimally inexpensive viscoelastic technique. Geophysics, 60(1), 176-184. https://doi.org/10.1190/1.1443744

Braga, I. L. S., and Moraes, F. S. (2013). High-resolution gathers by inverse $Q$ filtering in the wavelet domain. Geophysics, 78(2), V53-V61. https://doi.org/10.1190/geo2011-0508.1

Brzostowski, M. A., and McMechan, G. A. (1992). 3-D tomographic imaging of near-surface seismic velocity and attenuation. Geophysics, 57(3), 396-403. https://doi.org/10.1190/1.1443254

Carcione, J. M., Kosloff, D., and Kosloff, R. (1988). Wave propagation simulation in a linear viscoacoustic medium. Geophys. J. Int., 93(2), 393-401.
https://doi.org/10.1111/j.1365-246X.1988.tb02010.x

Carcione, J. M., Cavallini, F., Mainardi, F., and Hanyga, A. (2002). Time-domain modeling of constant- $Q$ seismic waves using fractional derivatives. Pure Appl. Geophys., 159(7), 1719-1736. https://doi.org/10.1007/s00024-0028705-z

Chang, W. F., and McMechan, G. A. (1994). 3-D elastic prestack, reverse-time depth migration. Geophysics, 59(4), 597-609. https://doi.org/10.1190/1.1443620

Chen, S. Q., Wei, Q., Liu, L. B., and Li, X. Y. (2018). Data-driven attenuation compensation via a shaping regularization scheme. IEEE Geosci. Remote Sens. Lett., 15(11), 1667-1671. https://doi.org/10.1109/LGRS.2018.2854731

Chernov, L. A. (1960). Wave Propagation in a Random Medium (Silverman, R. A., trans.). New York: McGraw-Hill.

Dasgupta, R., and Clark, R. A. (1998). Estimation of $Q$ from surface seismic reflection data. Geophysics, 63(6), 2120-2128. https://doi.org/10.1190/1.1444505

Deng, F., and McMechan, G. A. (2007). True-amplitude prestack depth migration. Geophysics, 72(3), S155-S166. https://doi.org/10.1190/1.2714334

Ding, Y., Du, Q. Z., Liu, L. H., and Zhang, Q. (2019). Feature analysis and compensation of seismic low-frequency based on compressed sensing and broad-band Yu-type low-passing shaping filter. Chin. J. Geophys. (in Chinese), 62(6), 2267-2275. https://doi.org/10.6038/cjg2019M0096

Dix, C. H. (1955). Seismic velocities from surface measurements. Geophysics, 20(1), 68-86. https://doi.org/10.1190/1.1438126

Du, Q. Z., Zhu, Y. T., and Ba, J. (2012). Polarity reversal correction for elastic reverse time migration. Geophysics, 77(2), S31-S41. https://doi.org/10.1190/geo2011-0348.1

Du, Q. Z., Guo, C. F., Zhao, Q., Gong, X. F., Wang, C. X., and Li, X. Y. (2017). Vectorbased elastic reverse time migration based on scalar imaging condition. Geophysics, 82(2), S111-S127. https://doi.org/10.1190/geo2016-0146.1

Dutta, G., and Schuster, G. T. (2016). Wave-equation $Q$ tomography. Geophysics, 81(6), R471-R484. https://doi.org/10.1190/geo2016-0081.1

Fomel, S. (2007). Shaping regularization in geophysical-estimation problems Geophysics, 72(2), R29-R36. https://doi.org/10.1190/1.2433716

Futterman, W. I. (1962). Dispersive body waves. J. Geophys. Res., 67(13), 5279-5291. https://doi.org/10.1029/JZ067i013p05279

Guo, P., McMechan, G. A., and Guan, H. M. (2016). Comparison of two viscoacoustic propagators for $Q$-compensated reverse time migration. Geophysics, 81(5), S281-S297. https://doi.org/10.1190/geo2015-0557.1

Hale, D. (1981). An inverse Q-filter. Stanford Exploration Project 26. 231-244. http://sep.stanford.edu/data/media/public/oldreports/oldreports/sep26/ 26_22.ps.gz

Hale, D. (1982). Q-adaptive deconvolution. In Proceedings of 1982 SEG Annual Meeting (pp. 82-83). Dallas, Texas: Society of Exploration Geophysicists. https://doi.org/10.1190/1.1827164

Hargreaves, N. D., and Calvert, A. J. (1991). Inverse $Q$ filtering by Fourier transform. Geophysics, 56(4), 519-527. https://doi.org/10.1190/1.1443067

Hestenes, M. R., and Steifel, E. (1952). Methods of conjugate gradients for solving linear systems. J. Res. Natl. Bur. Stand., 49(6), 409-436. https://doi.org/10.6028/jres.049.044

Julian, B. R., Gubbins, D. (1977). Three-dimensional seismic ray tracing. J. Geophys., 43(1), 95-113. https://journal.geophysicsjournal.com/JofG/article/view/133

Kamei, R., and Pratt, R. G. (2008). Waveform tomography strategies for imaging attenuation structure with cross-hole data. In Proceedings of the 70th EAGE Conference and Exhibition Incorporating SPE EUROPEC 2008. Rome, Italy, EAGE. https://doi.org/10.3997/2214-4609.20147680

Kamei, R., and Pratt, R. G. (2013). Inversion strategies for visco-acoustic waveform inversion. Geophys. J. Int., 194(2), 859-884. https://doi.org/10.1093/gji/ggt109

Kjartansson, E. (1979). Constant Q-wave propagation and attenuation. J. Geophys. Res., 84(B9), 4737-4748. https://doi.org/10.1029/JB084iB09p04737 Kolsky, H. (1953). Stress Waves in Solids. Oxford: Clarendon Press.

Kolsky, H. (1956). LXXI. The propagation of stress pulses in viscoelastic solids. Philos. Mag., 1(8), 693-710. https://doi.org/10.1080/14786435608238144

Li, G. F., Liu, Y., Zheng, H., and Huang, W. (2015). Absorption decomposition and compensation via a two-step scheme. Geophysics, 80(6), V145-V155. https://doi.org/10.1190/geo2015-0038.1

Li, Q. Z. (1993). A Systematical Analysis of High Resolution Seismic Exploration (in 
Chinese). Beijing: Petroleum Industry Press.

Li, Q. Z. (2017). High-Resolution Seismic Exploration. Tulsa, Oklahoma: Society of Exploration Geophysicists.

Liao, O. B., and McMechan, G. A. (1995). 2.5D full-wavefield viscoacoustic inversion. Geophys. Prospect., 43(8), 1043-1059. https://doi.org/10.1111/j.1365-2478.1995.tb00295.x

Liu, Y. S., Xu, T., Wang, Y. H., Teng, J. W., Badal, J., and Lan, H. Q. (2019). An efficient source wavefield reconstruction scheme using single boundary layer values for the spectral element method. Earth Planet. Phys., 3(4), 342-357. https://doi.org/10.26464/epp2019035

Margrave, G. F., Lamoureux, M. P., and Henley, D. C. (2011). Gabor deconvolution: estimating reflectivity by nonstationary deconvolution of seismic data. Geophysics, 76(3), W15-W30.

https://doi.org/10.1190/1.3560167

Margrave, G. F., Lamoureux, M. P., Grossman, J. P., and Iliescu, V. (2002). Gabor deconvolution of seismic data for source waveform and $\mathrm{Q}$ correction. In Proceedings of 2002 SEG Annual Meeting (pp. 2190-2193). Salt Lake City, Utah: Society of Exploration Geophysicists.

https://doi.org/10.1190/1.1817142

Mavko, G., and Nur, A. (1975). Melt squirt in the asthenosphere. J. Geophys. Res., 80(11), 1444-1448. https://doi.org/10.1029/JB080i011p01444

Mittet, R., Sollie, R., and Hokstad, K. (1995). Prestack depth migration with compensation for absorption and dispersion. Geophysics, 60(5), 1485-1494. https://doi.org/10.1190/1.1443882

Quan, Y. L., and Harris, J. M. (1997). Seismic attenuation tomography using the frequency shift method. Geophysics, 62(3), 895-905.

https://doi.org/10.1190/1.1444197

Rao, Y., and Wang, Y. H. (2019). Stabilised inverse- $Q$ filtering for seismic characterisation of carbonate reservoirs. J. Geophys. Eng., 16(1), 190-197. https://doi.org/10.1093/jge/gxy016

Robertsson, J. O., Blanch, J. O., and Symes, W. W. (1994). Viscoelastic finitedifference modeling. Geophysics, 59(9), 1444-1456. https://doi.org/10.1190/1.1443701

Shen, Y., Biondi, B., Clapp, R., and Nichols, D. (2014). Wave-equation migration $\mathrm{Q}$ analysis (WEMQA). In Proceedings of 2014 SEG Annual Meeting (pp. 3757-3762). Denver, Colorado: Society of Exploration Geophysicists. https://doi.org/10.1190/segam2014-1557.1

Shen, Y., Biondi, B., and Clapp, R. (2018). Q-model building using one-way wave-equation migration $Q$ analysis-Part 1: theory and synthetic test. Geophysics, 83(2), S93-S109. https://doi.org/10.1190/geo2016-0658.1

Shi, X. C., Mao, W. J., and Li, X. L. (2019). Viscoelastic Q-compensated Gaussian beam migration based on vector-wave imaging. Chin. J. Geophys., 64(4), 1480-1491. https://doi.org/10.6038/cjg2019L0797

Stolt, R. H. (1978). Migration by Fourier transform. Geophysics, 43(1), 23-48. https://doi.org/10.1190/1.1440826

Sun, J. Z., Zhu, T. Y., and Fomel, S. (2015). Viscoacoustic modeling and imaging using low-rank approximation. Geophysics, 80(5), A103-A108. https://doi.org/10.1190/geo2015-0083.1

Tikhonov, A. N. (1963). Solution of incorrectly formulated problems and the regularization method. Soviet Math. Dokl., 4, 1035-1038. https://ci.nii.ac.jp/naid/10006420921/

Tonn, R. (1991). The determination of the seismic quality factor Q from VSP data: a comparison of different computational methods. Geophys. Prospect., 39(1), 1-27. https://doi.org/10.1111/j.1365-2478.1991.tb00298.x

Traynin, P., Liu, J., and Reilly, J. M. (2008). Amplitude and bandwidth recovery beneath gas zones using Kirchhoff prestack depth Q-migration. In Proceedings of 2008 SEG Annual Meeting (pp. 2412-2416). Las Vegas, Nevada: Society of Exploration Geophysicists. https://doi.org/10.1190/1.3059363

Treeby, B. E., and Cox, B. T. (2010). Modeling power law absorption and dispersion for acoustic propagation using the fractional Laplacian. J. Acoust. Soc. Am., 127(5), 2741-2748. https://doi.org/10.1121/1.3377056

Varela, C. L., Rosa, A. L. R., and Ulrych, T. J. (1993). Modeling of attenuation and dispersion. Geophysics, 58(8), 1167-1173. https://doi.org/10.1190/1.1443500

Wang, B. F., Chen, X. H., Li, J. Y., Chen, Z. B., and Liu, G. C. (2015). A stable and efficient attenuation compensation method based on inversion. Chin. J. Geophys., 58(4), 375-386. https://doi.org/10.1002/cjg2.20181
Wang, B. F., and Lu, W. K. (2018). An efficient amplitude-preserving generalized $S$ transform and its application in seismic data attenuation compensation. IEEE Trans. Geosci. Remote Sens., 56(2), 859-866. https://doi.org/10.1109/TGRS.2017.2755666

Wang, S. D. (2011). Attenuation compensation method based on inversion. Appl. Geophys., 8(2), 150-157. https://doi.org/10.1007/s11770-011-0275-3

Wang, S. D., and Chen, X. H. (2014). Absorption-compensation method by $I_{1}$ norm regularization. Geophysics, 79(3), V107-V114. https://doi.org/10.1190/geo2013-0206.1

Wang, Y. F., Ma, X., Zhou, H., and Chen, Y. K. (2018). $L_{1-2}$ minimization for exact and stable seismic attenuation compensation. Geophys. J. Int., 213(3), 1629-1646. https://doi.org/10.1093/gji/ggy064

Wang, Y. H. (2002). A stable and efficient approach of inverse $Q$ filtering. Geophysics, 67(2), 657-663. https://doi.org/10.1190/1.1468627

Wang, Y. H. (2003). Quantifying the effectiveness of stabilized inverse $Q$ filtering. Geophysics, 68(1), 337-345. https://doi.org/10.1190/1.1543219

Wang, Y. H. (2004). Q analysis on reflection seismic data. Geophys. Res. Lett., 31(17), L17606. https://doi.org/10.1029/2004GL020572

Wang, Y. H., and Guo, J. (2004a). Modified Kolsky model for seismic attenuation and dispersion. J. Geophys. Eng., 1(3), 187-196. https://doi.org/10.1088/1742-2132/1/3/003

Wang, Y. H., and Guo, J. (2004b). Seismic migration with inverse $Q$ filtering. Geophys. Res. Lett., 31(21), L21608. https://doi.org/10.1029/2004GL020525

Wang, Y. H. (2006). Inverse $Q$-filter for seismic resolution enhancement. Geophysics, 71(3), V51-V60. https://doi.org/10.1190/1.2192912

Wang, Y. H. (2008). Seismic Inverse Q Filtering. Singapore: Blackwell Publishing.

Xie, Y., Xin, K. F., Sun, J., Notfors, C., Biswal, A. K., and Balasubramaniam, M. K. (2009). 3D prestack depth migration with compensation for frequency dependent absorption and dispersion. In Proceedings of 2009 SEG Annual Meeting (pp. 2919-2923). Houston, Texas: Society of Exploration Geophysicists. https://doi.org/10.1190/1.3255457

Xu, Y., and Chopra, S. (2007). Improving AVO fidelity by NMO stretching and offset-dependent tuning corrections. Lead. Edge, 26(12), 1548-1551. https://doi.org/10.1190/1.2821941

Xue, Y. J., Cao, J. X., and Wang, X. J. (2019). Inverse $Q$ filtering via synchrosqueezed wavelet transform. Geophysics, 84(2), V121-V132. https://doi.org/10.1190/geo2018-0177.1

Yan, H. Y., and Liu, Y. (2009). Estimation of $Q$ and inverse $Q$ filtering for prestack reflected PP- and converted PS-waves. Appl. Geophys., 6(1), 59-69. https://doi.org/10.1007/s11770-009-0009-y

Yilmaz, Ö. (2001). Seismic Data Analysis: Processing, Inversion, and Interpretation of Seismic Data. Tulsa, Oklahoma: Society of Exploration Geophysicists.

Yuan, S. Y., Wang, S. X., Ma, M., Ji, Y. Z., and Deng, L. (2017). Sparse Bayesian learning-based time-variant deconvolution. IEEE Trans. Geosci. Remote Sens., 55(11), 6182-6194. https://doi.org/10.1109/TGRS.2017.2722223

Zhang, B. L., Ni, S. D., and Chen, Y. L. (2019). Seismic attenuation in the lower mantle beneath Northeast China constrained from short-period reflected core phases at short epicentral distances. Earth Planet. Phys., 3(6), 537-546. https://doi.org/10.26464/epp2019055

Zhang, C. J., and Ulrych, T. J. (2007). Seismic absorption compensation: a least squares inverse scheme. Geophysics, 72(6), R109-R114. https://doi.org/10.1190/1.2766467

Zhang, G. W., and Gao, J. H. (2018). Inversion-driven attenuation compensation using synchrosqueezing transform. IEEE Geosci. Remote Sens. Lett., 15(1), 132-136. https://doi.org/10.1109/LGRS.2017.2777598

Zhang, J. F., and Wapenaar, K. (2002). Wavefield extrapolation and prestack depth migration in anelastic inhomogeneous media. Geophys. Prospect., 50(6), 629-643. https://doi.org/10.1046/j.1365-2478.2002.00342.x

Zhang, J. F., Wu, J. Z., and Li, X. Y. (2013). Compensation for absorption and dispersion in prestack migration: an effective $Q$ approach. Geophysics, 78(1), S1-S14. https://doi.org/10.1190/geo2012-0128.1

Zhang, Y., Zhang, P., and Zhang, H. Z. (2010). Compensating for visco-acoustic effects in reverse-time migration. In Proceedings of 2010 SEG Annual Meeting (pp. 3160-3164). Denver, Colorado: Society of Exploration Geophysicists. https://doi.org/10.1190/1.3513503

Zhu, T. Y., and Harris, J. M. (2014). Modeling acoustic wave propagation in heterogeneous attenuating media using decoupled fractional Laplacians. Geophysics, 79(3), T105-T116. https://doi.org/10.1190/geo2013-0245.1 


\section{Supplementary Materials for "Seismic attenuation compensation with spectral-shaping regularization"}

\section{Offset-Related Q Filters Based on the Modified Kolsky Model}

On the basis of the demonstration of seismic ray propagation in Figure 1, backward wavefield continuation of plane waves in the frequency domain can be expressed as

$$
\boldsymbol{U}\left(T+\Delta T_{i}, \omega\right)=\boldsymbol{U}(T, \omega) \mathrm{e}^{j k(\omega) v_{r} \Delta T_{i}},
$$

where $\boldsymbol{U}(T, \omega)$ denotes a wavefield with propagating time $T$ and angular frequency $\omega, \Delta T_{i}$ is the travel time increment of seismic waves in layer $i, k(\omega)$ is the frequency-dependent wavenumber, and $v_{r}$ is the velocity of reference frequency. Variable $j$ denotes an imaginary unit. According to the geometry in Figure 1 and Snell's law, $\Delta T_{i}$ can be represented as

$$
\Delta T_{i}=\frac{2 h_{i}}{v_{i} \cos \theta_{i}}=\frac{\Delta T_{i, 0}}{\cos \theta_{i}} .
$$

The $\Delta T_{i, 0}$ in Equation (A2) is the two-way travel time increment of seismic wave propagation vertically in layer $i$, and $p$ denotes the ray parameter. Under the assumption of a horizontally stratified medium, incident angle $\theta_{i}$ of seismic rays in each layer $i$ is computationally available through the ray-tracing technique (Julian and Gubbins, 1977; Yan HY and Liu Y, 2009) with a given offset $x$.

Substituting Equation (A2) into Equation (A1), wavefield continuation is then written as

$$
\boldsymbol{U}\left(T+\Delta T_{i}, \omega\right)=\boldsymbol{U}(T, \omega) \mathrm{e}^{j k(\omega) v_{r} \frac{\Delta T_{i, 0}}{\cos \theta_{i}}} .
$$

We used a modified Kolsky model (Wang YH and Guo J, 2004a) to characterize the velocity dispersion and seismic attenuation. Thus, the backward wavefield continuation (inverse-Q filter) can be expressed as

$$
\boldsymbol{U}\left(T+\Delta T_{i}, \omega\right)=\boldsymbol{U}(T, \omega) \mathrm{e}^{j \omega\left(\frac{\omega}{\omega_{h}}\right)^{-\gamma_{i}} \frac{\Delta T_{i, 0}}{\cos \theta_{i}}} \mathrm{e}^{\left(\frac{\omega}{\omega_{h}}\right)^{-\gamma_{i}} \frac{\omega}{2 Q_{i}} \frac{\Delta T_{i, 0}}{\cos \theta_{i}},}
$$

where the tuning frequency $\omega_{h}$ is the highest possible seismic frequency (Wang $\mathrm{YH}$ and Guo J, 2004a) and $\gamma_{i}=\frac{1}{\pi} \tan ^{-1} \frac{1}{Q} \approx \frac{1}{\pi Q_{i}}$ (Kjartansson, 1979). In contrast to conventional zero-offset $Q$ filters (Wang YH, 2002), Equation (A4) includes the cosine of each in- cident angle in both the exponential operators, thus making the wavefield continuation related to the offset.

For seismic wave propagation through $n$ layers, the inverse- $Q$ filter becomes

$$
\boldsymbol{U}\left(T+\sum_{i=1}^{n} \Delta T_{i}, \omega\right)=\boldsymbol{U}(T, \omega) \mathrm{e}^{j \sum_{i=1}^{n}\left[\omega\left(\frac{\omega}{\omega_{h}}\right)^{-\gamma_{i}} \frac{\Delta T_{i, 0}}{\cos \theta_{i}}\right]} \mathrm{e}^{\sum_{i=1}^{n}\left[\left(\frac{\omega}{\omega_{h}}\right)^{-\gamma_{i}} \frac{\omega}{2 Q_{i}} \frac{\Delta T_{i, 0}}{\cos \theta_{i}}\right] .}
$$

In actual computation, travel time $\Delta T_{i, 0}$ is substituted by the sampling interval $d t$ and the continuous frequency $\omega$ is discretized as $\omega_{m}$. Therefore, Equation (A5) will be rewritten as

$$
\boldsymbol{U}(T+n \cdot \mathrm{d} t, \omega)=\boldsymbol{U}(T, \omega) \mathrm{e}^{j \sum_{i=1}^{n}\left[\omega_{m}\left(\frac{\omega_{m}}{\omega_{h}}\right)^{\gamma_{i}} \frac{\mathrm{d} t}{\cos \theta_{i}}\right]} \mathrm{e}^{-\sum_{i=1}^{n}\left[\left(\frac{\omega_{m}}{\omega_{h}}\right)^{-\gamma_{i}} \frac{\omega_{m}}{2 Q_{i}} \frac{\mathrm{d} t}{\cos \theta_{i}}\right] .}
$$

An extra $\cos \theta_{i}$ is included in the offset-related $\mathrm{Q}$ filters, making it difficult to compute recursively (Wang $\mathrm{YH}, 2002$ ). The medium through which seismic waves propagate can be considered approximately homogeneously equivalent. On such an approximation, the interval quality factor of the first $n$ layers will be represented by effective quality factor $Q_{e, n}$ (Bickel and Natarajan, 1985) as

$$
\frac{T_{n-1}+\frac{\mathrm{d} t}{\cos \theta_{n}}}{Q_{e, n}}=\frac{T_{n-1}}{Q_{e, n-1}}+\frac{\frac{\mathrm{d} t}{\cos \theta_{n}}}{Q_{n}},
$$

where $Q_{n}$ denotes the interval Q of layer $n$, and $T_{n}$ is the travel time cumulation in $n$ layers. Consequently, Equation (A7) can be further approximated as

$\left.\boldsymbol{U}(T+n \cdot \mathrm{d} t, \omega) \approx \boldsymbol{U}(T, \omega) \mathrm{e}^{j\left[\omega_{m}\left(\frac{\omega_{m}}{\omega_{h}}\right)^{-\gamma_{e, n}} \sum_{i=1}^{n} \frac{\mathrm{d} t}{\cos \theta_{i}}\right]}\right]_{\mathrm{e}}^{\left[\left(\frac{\omega_{m}}{\omega_{h}}\right)^{-\gamma_{e, n}} \frac{\omega_{m}}{2 Q_{e, n}} \sum_{i=1}^{n} \frac{\mathrm{d} t}{\cos \theta_{i}}\right] .}$

As the reverse of inverse- $Q$ filtering, forward- $Q$ filtering can completely decompensate the effects of the inverse-Q filter with the same $\mathrm{Q}$ model. According to the zero-offset forward-Q filter given by Wang YH (2008), the offset-related forward-Q filter can be written as

$\boldsymbol{U}(T+n \cdot \mathrm{d} t, \omega) \approx \boldsymbol{U}(T, \omega) \mathrm{e}^{\left[\omega_{m}\left(\frac{\omega_{m}}{\omega_{h}}\right)^{\gamma_{e, n}} \sum_{i=1}^{n} \frac{\mathrm{d} t}{\cos \theta_{i}}\right]} \mathrm{e}^{\left[\left(\frac{\omega_{m}}{\omega_{h}}\right)^{-\gamma_{e, n}} \frac{\omega_{m}}{2 Q_{e, n}} \sum_{i=1}^{n} \frac{\mathrm{d} t}{\cos \theta_{i}}\right]}$. 